Part of Journal of Research of the National Bureau of Standards, Volume 35, July 1945

\title{
SINGLE-CYLINDER ENGINE TESTS OF SUBSTITUTE MOTOR FUELS
}

\author{
By Donald B. Brooks
}

\section{ABSTRACT}

Single-cylinder-engine tests of nonhydrocarbon fuels and gasoline, at fixed compression ratio and at the compression ratio for trace knock for each fuel, show no material differences in performance other than those associated with differences in heats of combustion and vaporization. All the nonhydrocarbon fuels could be operated at compression ratios higher than was permissible with the gasoline, with corresponding increases in power and thermal efficiency.

\section{CONTENTS}

Page

I. Scope of investigation,
II. Fuels, test equipment, and procedure

III. Test results

IV. Discussion of test results

1. Power at constant compression ratio $\ldots \ldots$

2. Comparison of power with calculated values $\ldots \ldots \ldots$

3. Optimum spark advance

4. Power at compression ratio for trace knock $\ldots \ldots$

5. Combustion performance of fuels

(a) Relative power versus heat input 29

(b) Rate of burning

(c) Thermal-plug temperatures

6. Power at constant spark advance

7. Power-mixture-spark-advance relationship

V. Conclusions

VI. Appendix

\section{SCOPE OF INVESTIGATION}

The work reported herein is a portion of an extensive investigation of substitute motor fuels conducted by the National Bureau of Standards for the United States Foreign Economic Administration. The Bureau's part in this investigation, begun in 1942, was to develop technical information on the utilization of substitute fuels that could be produced in foreign countries where petroleum products were scarce and where military exigencies required the use of automotive transport. Considerations of economics and production specifically were not part of the investigation as assigned to this Bureau, although 
it was understood that no data were to be developed on materials the production of which would involve unreasonable costs.

The objective of the work covered by this report was to determine, on a precision test engine, the power, thermal efficiency, and combustion performance of each of a series of substitute fuels and of gasoline, both at the compression ratio giving trace knock on gasoline and at the compression ratio at which trace knock prevailed with each fuel.

\section{FUELS, TEST EQUIPMENT, AND PROCEDURE}

The fuels used in this work were selectèd from those used in the liquid-fuels phase of the major investigation, and included only nonhydrocarbon fuels, with the exception of the reference gasoline. Table 1 gives the composition, knock rating, and experimental heat of combustion as determined by bomb calorimeter of each fuel used in this part of the work. The heat of combustion values were determined by R. S. Jessup of this Bureau, and are believed to be accurate to 0.1 percent.

TABLE 1.-Data on test fuels

\begin{tabular}{|c|c|c|c|c|c|}
\hline \multirow{2}{*}{ Fuel } & \multirow{2}{*}{ Composition } & \multicolumn{2}{|c|}{$\begin{array}{l}\text { Heat of combuss- } \\
\text { tion, }{ }^{\circ} \text { Btu/lb at } \\
\text { at } 30^{\circ}\end{array}$} & \multicolumn{2}{|c|}{ Octane number } \\
\hline & & Higher & Lower & $\begin{array}{c}\text { ASTM } \\
\text { motor }\end{array}$ & $\begin{array}{l}\mathrm{CFR} \\
\text { research }\end{array}$ \\
\hline $\begin{array}{l}\text { B } \\
\text { C } \\
\text { D } \\
\end{array}$ & $\begin{array}{l}\text { Reference gasoline } \\
\text { 190-proof ethyl alcohol } \\
200 \text {-proof ethyl alcohol } \\
75 \text { percent ethanol, } 25 \text { percent diethyl ether } \\
\text { Reference gasoline. } \\
50 \text { percent acetone, } 50 \text { percent butanol } \\
27 \text { percent acetone, } 6 \text { percent ethanol, } 67 \text { percent }\end{array}$ & $\begin{array}{l}(\mathrm{a}) \\
11,760 \\
12,700 \\
13,410 \\
20,170 \\
14,380 \\
14,650\end{array}$ & $\begin{array}{l}(\mathbf{s}) \\
10,570 \\
11,520 \\
12,850 \\
18,810 \\
13,270 \\
13,500\end{array}$ & $\begin{array}{l}70 \\
94 \\
92 \\
89 \\
70 \\
90 \\
88\end{array}$ & $\begin{array}{l}72 \\
\text { a } 2.7 \\
\text { a } 1.4 \\
\text { b } 0.5 \\
72 \\
\text { b } 0.2 \\
\text { b. }\end{array}$ \\
\hline 3. & $\begin{array}{l}\text { butanol. } \\
\text { 28.5 percent acetone, } 71.5 \text { percent butanol }\end{array}$ & 14,860 & 13,690 & 87 & 100 \\
\hline
\end{tabular}

a Assumed to be same as for fuel $\mathrm{E}$.

b Milliliters of tetraethyl lead per gallon of isooctane.

The test apparatus consisted of a CFR (Coordinating Fuel Research Committee) single-cylinder variable-compression engine direct-connected to an essentially constant-speed alternating-current dynamometer, and provided with all necessary test instruments. Fuel was injected into the intake pipe, the stroke of the injector being controlled with a micrometer screw. Spark advance was indicated by the capacitance discharge of a neon tube mounted in an insulating disk attached to the crankshaft. The temperature of a thermal plug screwed into the cylinder head was shown on an automaticindicating potentiometer graduated in degrees Fahrenheit. As the engine is in effect hopper-cooled, coolant temperature is automatically maintained at the boiling point. Fuel consumption was determined by noting the time required to use one-fourth pound of fuel.

With the engine operating at best-power mixture ratio and optimum spark advance on gasoline (reference fuel blend) of 70 motor octane number, the compression ratio was adjusted to give trace knock. The ratio so found was 5.11. Each fuel was tested under this condition; each fuel was also tested under the compression ratio at which the fuel itself gave trace knock at best-power mixture ratio and optimum spark advance. 
The same test procedure was followed with each fuel. The fuel flow was adjusted initially to approximately the least rate at which consistent firing could be obtained. While operating at this rate, two or more measurements of fuel consumption were made. Coincidentally, observations of power and of thermal-plug temperature were made at a series of spark settings from full retard to full advance, a range of about 40 degrees. At the conclusion of these readings, the fuel flow was increased and the observations were repeated. This was continued, in appropriate steps, to a fuel flow about 50 percent greater than that giving maximum power. Readings of barometric pressure, ambient temperature, atmospheric humidity, and engine friction were taken frequently throughout the tests.

Power and fuel-consumption rates were corrected to standard conditions of $59^{\circ} \mathrm{F}$. and $29.53 \mathrm{in}$. $\mathrm{Hg}$ dry air pressure by the formula

where

$$
P_{s}=P_{0} \frac{29.53}{B-h} \sqrt{\frac{460+t}{519}}
$$

$P_{0}=$ observed power or fuel consumption

$P_{s}=$ corrected power or fuel consumption

$B=$ barometric pressure, in. $\mathrm{Hg}$

$h=$ pressure of water vapor, in. $\mathrm{Hg}$

$t=$ intake air temperature, ${ }^{\circ} \mathrm{F}$.

\section{TEST RESULTS}

The sum of the brake and friction power readings at each rate of fuel flow was plotted against the respective spark advance. A sample of the resulting curves is shown in figure 15. The coordinates of the peak of each curve were determined and tabulated, together with the faired values of indicated power at each 5 degrees of spark advance. These values are given in tables 2 to 15 .

TABLE 2.-Power and economy tests on reference gasoline-first run

The lower set of data was obtained on a different day from the upper set

[Compression ratio 5.11]

\begin{tabular}{|c|c|c|c|c|c|c|c|c|c|c|c|}
\hline \multirow{2}{*}{$\begin{array}{l}\text { Fuel } \\
\text { con- } \\
\text { sump- } \\
\text { tion }\end{array}$} & \multirow{2}{*}{$\begin{array}{c}\text { Fuel heat } \\
\text { input }\end{array}$} & \multicolumn{7}{|c|}{ Indicated horsepower at spark advance of- } & \multirow{2}{*}{$\begin{array}{l}\text { Maxi- } \\
\text { mum } \\
\text { indi- } \\
\text { cated } \\
\text { horse } \\
\text { power }\end{array}$} & \multirow{2}{*}{$\begin{array}{c}\text { Opti- } \\
\text { mum } \\
\text { spark } \\
\text { advance }\end{array}$} & \multirow{2}{*}{$\begin{array}{l}\text { Specific } \\
\text { fuel con- } \\
\text { sump- } \\
\text { tion }\end{array}$} \\
\hline & & $40^{\circ}$ & $45^{\circ}$ & $50^{\circ}$ & $55^{\circ}$ & $60^{\circ}$ & $65^{\circ}$ & $70^{\circ}$ & & & \\
\hline $\begin{array}{c}l b / h r \\
5.66 \\
5.98 \\
6.49 \\
6.75 \\
7.28\end{array}$ & $\begin{array}{c}\text { Btu/hr } \times \\
10^{-9} \\
114.2 \\
120.6 \\
130.9 \\
136.1 \\
146.8\end{array}$ & $\begin{array}{l}12.08 \\
12.42 \\
12.65 \\
12.64 \\
12.55\end{array}$ & $\begin{array}{l}12.34 \\
12.64 \\
12.78 \\
12.78 \\
12.63\end{array}$ & $\begin{array}{l}12.46 \\
12.71 \\
12.79 \\
12.80 \\
12.64\end{array}$ & $\begin{array}{l}12.48 \\
12.71 \\
12.73 \\
12.68 \\
12.57\end{array}$ & $\begin{array}{l}12.45 \\
12.65 \\
12.62 \\
12.49 \\
12.42\end{array}$ & $\begin{array}{l}12.36 \\
12.54 \\
12.45 \\
12.27 \\
12.19\end{array}$ & $\begin{array}{l}12.25 \\
12.38 \\
12.24 \\
12.00 \\
11.93\end{array}$ & $\begin{array}{l}12.49 \\
12.72 \\
12.80 \\
12.80 \\
12.64\end{array}$ & $\begin{array}{r}\text { Degrees } \\
53.9 \\
52.2 \\
49.0 \\
47.7 \\
47.6\end{array}$ & $\begin{array}{r}\text { lb/ihp- } \\
h r \\
0.453 \\
.470 \\
.507 \\
.527 \\
.576\end{array}$ \\
\hline $\begin{array}{r}5.21 \\
5.98 \\
6.56 \\
6.83 \\
7.84 \\
8.56 \\
9.43 \\
10.18\end{array}$ & $\begin{array}{l}105.1 \\
120.6 \\
132.3 \\
137.8 \\
158.1 \\
172.7 \\
190.2 \\
205.3\end{array}$ & $\begin{array}{r}9.90 \\
12.31 \\
12.42 \\
12.54 \\
12.38 \\
12.04 \\
11.60 \\
10.94\end{array}$ & $\begin{array}{l}10.39 \\
12.49 \\
12.54 \\
12.68 \\
12.51 \\
12.26 \\
11.90 \\
11.32\end{array}$ & $\begin{array}{l}10.76 \\
12.58 \\
12.58 \\
12.72 \\
12.56 \\
12.40 \\
12.09 \\
11.60\end{array}$ & \begin{tabular}{l|}
10.81 \\
12.59 \\
12.55 \\
12.69 \\
12.50 \\
12.36 \\
12.12 \\
11.74
\end{tabular} & $\begin{array}{l}10.70 \\
12.56 \\
12.46 \\
12.59 \\
12.37 \\
12.19 \\
12.03 \\
11.78\end{array}$ & $\begin{array}{l}10.55 \\
12.49 \\
12.33 \\
12.44 \\
12.17 \\
11.96 \\
11.88 \\
11.77\end{array}$ & $\begin{array}{l}10.38 \\
12.39 \\
12.14 \\
12.25 \\
11.83 \\
11.69 \\
11.65 \\
11.70\end{array}$ & $\begin{array}{l}10.83 \\
12.59 \\
12.58 \\
12.72 \\
12.56 \\
12.42 \\
12.12 \\
11.78\end{array}$ & $\begin{array}{l}53.0 \\
54.5 \\
50.6 \\
50.6 \\
50.0 \\
51.0 \\
53.7 \\
61.1\end{array}$ & $\begin{array}{l}.481 \\
.475 \\
.521 \\
.537 \\
.624 \\
.689 \\
.778 \\
.864\end{array}$ \\
\hline \multicolumn{10}{|c|}{ Iaximum power...... 12.80 at $6.60 \mathrm{lb} / \mathrm{hr}$. Minimum values....... } & 47.5 & 0.452 \\
\hline
\end{tabular}




\section{Journal of Research of the National Bureau of Standards}

TABLE 3.-Power and economy tests on 190-proof ethyl alcohol

[Compression ratio 5.11]

\begin{tabular}{|c|c|c|c|c|c|c|c|c|c|c|c|c|}
\hline \multirow[b]{2}{*}{$\begin{array}{c}\text { Fuel } \\
\text { con- } \\
\text { sump- } \\
\text { tion }\end{array}$} & \multirow[b]{2}{*}{$\begin{array}{c}\text { Fuel } \\
\text { heat in- } \\
\text { put }\end{array}$} & \multicolumn{7}{|c|}{ Indicated horsepower at spark advance of- } & \multirow{2}{*}{$\begin{array}{l}\text { Maxi- } \\
\text { mum } \\
\text { indi- } \\
\text { cated } \\
\text { horse- } \\
\text { power }\end{array}$} & \multirow[b]{2}{*}{$\begin{array}{l}\text { Opti- } \\
\text { mum } \\
\text { spark } \\
\text { ad- } \\
\text { vance }\end{array}$} & \multirow[b]{2}{*}{$\begin{array}{l}\text { Specific } \\
\text { fuel con- } \\
\text { sump- } \\
\text { tion }\end{array}$} & \multirow{2}{*}{$\begin{array}{l}\text { Ther- } \\
\text { mometer } \\
\text { plug } \\
\text { temper- } \\
\text { ature at } \\
\text { opti- } \\
\text { mum } \\
\text { spark ad- } \\
\text { vance }\end{array}$} \\
\hline & & $40^{\circ}$ & $45^{\circ}$ & $50^{\circ}$ & $55^{\circ}$ & $60^{\circ}$ & $65^{\circ}$ & $70^{\circ}$ & & & & \\
\hline lb/hr & $\begin{array}{l}\text { Btu/hr } \\
\times 10^{-3}\end{array}$ & & & & & & & & & Degrees & $l b / i h p-h r$ & \\
\hline 8.18 & 96.2 & 8.65 & 9.10 & 9.47 & 9.76 & 9. 98 & 10.13 & 10.22 & 10.28 & 76.0 & 0.796 & $<600$ \\
\hline 8. 71 & 102.4 & 10.70 & 11.10 & 11.36 & 11. 52 & 11. & 11. & & & & .749 & 617 \\
\hline 9.93 & & 12 & & & & & & & & & & 711 \\
\hline 11. 10 & 130 & 1 & & 13. & 13. & & 13 & & & & & \\
\hline 12. 53 & 147. & 13. 29 & 13. & 13. 40 & 13. 29 & 13. & 12. & 12. & 13. & 47 & .934 & 726 \\
\hline 13. 87 & & & 13 & 13. & 13. 22 & 13. & & & & & & 684 \\
\hline 1. & & 12 & 12. & 13. & 13. 05 & 13. & & & 13 & & 1 & \\
\hline 16. 1 & 190. & 12.2 & 12. & 12. & 12.75 & 12. & 12. & & 12 & & $\begin{array}{l}1.107 \\
1.269\end{array}$ & 610 \\
\hline 16. 71 & 196. 5 & 11.62 & 12.12 & 12.31 & 12.41 & 12.44 & 12.44 & 12. 38 & 12.44 & 61.6 & 1. 343 & $<600$ \\
\hline \multicolumn{10}{|c|}{ Maximum power_._._13.46 at $11.40 \mathrm{lb} / \mathrm{l}$} & 47.0 & 0.746 & \\
\hline
\end{tabular}

TABLE 4.-Power and economy tests on 200-proof ethyl alcohol

[Compressed ratio 5.11]

\begin{tabular}{|c|c|c|c|c|c|c|c|c|c|c|c|c|}
\hline \multirow[b]{2}{*}{$\begin{array}{c}\text { Fuel } \\
\text { con- } \\
\text { sump- } \\
\text { tion }\end{array}$} & \multirow[b]{2}{*}{$\begin{array}{c}\text { Fuel } \\
\text { heat in- } \\
\text { put }\end{array}$} & \multicolumn{7}{|c|}{ Indicated horsepower at spark advance of- } & \multirow{2}{*}{$\begin{array}{l}\text { Maxi- } \\
\text { mumi } \\
\text { indi- } \\
\text { cated } \\
\text { horse- } \\
\text { power }\end{array}$} & \multirow[b]{2}{*}{$\begin{array}{l}\text { Opti- } \\
\text { mum } \\
\text { spark } \\
\text { ad- } \\
\text { vance }\end{array}$} & \multirow[b]{2}{*}{$\begin{array}{l}\text { Specific } \\
\text { fuel con- } \\
\text { sump- } \\
\text { tion }\end{array}$} & \multirow{2}{*}{$\begin{array}{l}\text { Ther- } \\
\text { mometer } \\
\text { plug } \\
\text { temper- } \\
\text { ature at } \\
\text { opti- } \\
\text { mum } \\
\text { spark ad- } \\
\text { vance }\end{array}$} \\
\hline & & $40^{\circ}$ & $45^{\circ}$ & $50^{\circ}$ & $55^{\circ}$ & $60^{\circ}$ & $65^{\circ}$ & $70^{\circ}$ & & & & \\
\hline $\begin{array}{r}l b / h r \\
7.58 \\
8.09 \\
8.76 \\
10.34 \\
11.59 \\
13.14 \\
14.59 \\
15.61 \\
16.08\end{array}$ & $\begin{array}{r}\text { Btu, hr } \\
\times 10^{-3} \\
96.4 \\
102.9 \\
111.4 \\
131.5 \\
147.4 \\
167.1 \\
185.6 \\
198.6 \\
204.5\end{array}$ & $\begin{array}{r}9.00 \\
10.78 \\
11.91 \\
13.27 \\
13.28 \\
13.18 \\
12.77 \\
12.30 \\
11.87\end{array}$ & $\begin{array}{r}9.44 \\
11.03 \\
12.20 \\
13.36 \\
13.37 \\
13.31 \\
13.04 \\
12.59 \\
12.23\end{array}$ & $\begin{array}{r}9.75 \\
11.25 \\
12.35 \\
13.37 \\
13.35 \\
13.32 \\
13.16 \\
12.76 \\
12.39\end{array}$ & $\begin{array}{r}9.96 \\
11.43 \\
12.41 \\
13.30 \\
13.23 \\
13.24 \\
13.15 \\
12.82 \\
12.45\end{array}$ & $\begin{array}{l}10.12 \\
11.56 \\
12.41 \\
13.16 \\
13.01 \\
13.07 \\
13.03 \\
12.84 \\
12.46\end{array}$ & $\begin{array}{l}10.20 \\
11.62 \\
12.35 \\
12.92 \\
12.74 \\
12.85 \\
12.87 \\
12.82 \\
12.44\end{array}$ & $\begin{array}{l}10.22 \\
11.63 \\
12.20 \\
12.60 \\
12.41 \\
12.60 \\
12.67 \\
12.76 \\
12.36\end{array}$ & $\begin{array}{l}10.22 \\
11.64 \\
12.42 \\
13.38 \\
13.37 \\
13.33 \\
13.16 \\
12.84 \\
12.46\end{array}$ & \begin{tabular}{|c|} 
Degrees \\
69.0 \\
67.4 \\
57.3 \\
48.0 \\
46.6 \\
48.0 \\
51.7 \\
60.5 \\
59.2
\end{tabular} & $\begin{array}{r}\text { lb/ihp-hr } \\
0.742 \\
.695 \\
.705 \\
.773 \\
.867 \\
.986 \\
1.109 \\
1.216 \\
1.291\end{array}$ & $\begin{array}{r}{ }^{\circ} F \\
<600 \\
<600 \\
681 \\
740 \\
723 \\
700 \\
654 \\
610 \\
<600\end{array}$ \\
\hline $100 x$ & 1 por & & 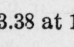 & 1 & ir. I & & & & & 46.9 & 0.695 & \\
\hline
\end{tabular}


TABLE 5.-Power and economy tests on ether blend, fuel D

[Compression ratio 5.11]

\begin{tabular}{|c|c|c|c|c|c|c|c|c|c|c|c|c|}
\hline \multirow[b]{2}{*}{$\begin{array}{l}\text { Fuel } \\
\text { con- } \\
\text { sump- } \\
\text { tion }\end{array}$} & \multirow[b]{2}{*}{$\begin{array}{c}\text { Fuel } \\
\text { heat in- } \\
\text { put }\end{array}$} & \multicolumn{7}{|c|}{ Indicated horsepower at spark advance of- } & \multirow[b]{2}{*}{$\begin{array}{l}\text { Maxi- } \\
\text { mum } \\
\text { indi- } \\
\text { cated } \\
\text { horse- } \\
\text { power }\end{array}$} & \multirow[b]{2}{*}{$\begin{array}{l}\text { Opti- } \\
\text { mum } \\
\text { spark } \\
\text { ad- } \\
\text { vance }\end{array}$} & \multirow[b]{2}{*}{$\begin{array}{c}\text { Specific } \\
\text { fuel con- } \\
\text { sump- } \\
\text { tion }\end{array}$} & \multirow{2}{*}{$\begin{array}{l}\text { Ther- } \\
\text { mometer } \\
\text { plug } \\
\text { temper- } \\
\text { ature at } \\
\text { opti- } \\
\text { mum } \\
\text { spark ad- } \\
\text { vance }\end{array}$} \\
\hline & & $40^{\circ}$ & $45^{\circ}$ & $50^{\circ}$ & $55^{\circ}$ & $60^{\circ}$ & $65^{\circ}$ & $70^{\circ}$ & & & & \\
\hline $\begin{array}{r}l b / h r \\
7.19 \\
7.60 \\
8.58 \\
9.45 \\
10.64 \\
11.60 \\
12.84 \\
13.81 \\
15.01 \\
15.77\end{array}$ & $\begin{array}{r}\text { Btu.hr } \\
\times 10^{-3} \\
96.4 \\
101.9 \\
115.1 \\
126.7 \\
142.7 \\
155.6 \\
172.2 \\
185.2 \\
201.3 \\
211.5\end{array}$ & $\begin{array}{r}8.58 \\
10.44 \\
12.49 \\
13.25 \\
13.35 \\
13.31 \\
13.12 \\
12.89 \\
12.27 \\
11.71\end{array}$ & $\begin{array}{r}9.23 \\
10.72 \\
12.72 \\
13.35 \\
13.40 \\
13.36 \\
13.24 \\
13.05 \\
12.59 \\
12.08\end{array}$ & $\begin{array}{r}9.62 \\
10.93 \\
12.84 \\
13.35 \\
13.36 \\
13.31 \\
13.23 \\
13.09 \\
12.78 \\
12.32\end{array}$ & $\begin{array}{r}9.88 \\
11.07 \\
12.84 \\
13.28 \\
13.23 \\
13.16 \\
13.13 \\
13.06 \\
12.86 \\
12.48\end{array}$ & $\begin{array}{l}10.05 \\
11.17 \\
12.76 \\
13.13 \\
13.02 \\
12.94 \\
12.92 \\
12.93 \\
12.85 \\
12.57\end{array}$ & $\begin{array}{l}10.14 \\
11.21 \\
12.61 \\
12.90 \\
12.68 \\
12.67 \\
12.68 \\
12.76 \\
12.78 \\
12.60\end{array}$ & $\begin{array}{l}10.13 \\
11.19 \\
12.41 \\
12.60 \\
12.31 \\
12.32 \\
12.39 \\
12.57 \\
12.67 \\
12.56\end{array}$ & $\begin{array}{l}10.15 \\
11.21 \\
12.85 \\
13.36 \\
13.40 \\
13.34 \\
13.25 \\
13.07 \\
12.86 \\
12.60\end{array}$ & $\begin{array}{c}\text { Degrees } \\
67.4 \\
66.2 \\
52.6 \\
47.4 \\
44.9 \\
44.6 \\
47.1 \\
50.0 \\
57.3 \\
64.8\end{array}$ & $\begin{array}{r}l b / i h p-h r \\
0.708 \\
.678 \\
.668 \\
.707 \\
.794 \\
.870 \\
.969 \\
1.057 \\
1.167 \\
1.252\end{array}$ & $\begin{array}{r}{ }^{\circ} F \\
<600 \\
612 \\
720 \\
755 \\
739 \\
716 \\
687 \\
662 \\
625 \\
616\end{array}$ \\
\hline$\omega_{2}$ & & & & & & & & & & 44.5 & 0.663 & \\
\hline
\end{tabular}

TABLE 6.-Power and economy tests on blend 1

[Compression ratio 5.11]

\begin{tabular}{|c|c|c|c|c|c|c|c|c|c|c|c|c|}
\hline \multirow[b]{2}{*}{$\begin{array}{l}\text { Fuel } \\
\text { con- } \\
\text { sump- } \\
\text { tion }\end{array}$} & \multirow[b]{2}{*}{$\begin{array}{c}\text { Fuel } \\
\text { heat in- } \\
\text { put }\end{array}$} & \multicolumn{7}{|c|}{ Indicated horsepower at spark advance of- } & \multirow[b]{2}{*}{$\begin{array}{l}\text { Maxi- } \\
\text { mum } \\
\text { indi- } \\
\text { cated } \\
\text { horse- } \\
\text { power }\end{array}$} & \multirow[b]{2}{*}{$\begin{array}{c}\text { Opti- } \\
\text { mum } \\
\text { spark } \\
\text { ad- } \\
\text { vance }\end{array}$} & \multirow[b]{2}{*}{$\begin{array}{c}\text { Specific } \\
\text { fuel con- } \\
\text { sump- } \\
\text { tion }\end{array}$} & \multirow{2}{*}{$\begin{array}{l}\text { Ther- } \\
\text { mometer } \\
\text { plug } \\
\text { temper- } \\
\text { ature at } \\
\text { opti- } \\
\text { mum } \\
\text { spark ad- } \\
\text { vance }\end{array}$} \\
\hline & & $40^{\circ}$ & $45^{\circ}$ & $50^{\circ}$ & $55^{\circ}$ & $60^{\circ}$ & $65^{\circ}$ & $70^{\circ}$ & & & & \\
\hline $\begin{array}{r}l b / h r \\
7.05 \\
7.65 \\
8.61 \\
10.30 \\
11.42 \\
12.41 \\
13.24 \\
14.32\end{array}$ & $\begin{array}{c}\text { Btu/hr } \\
\times 10^{-3} \\
101.4 \\
110.0 \\
123.8 \\
148.1 \\
164.2 \\
178.5 \\
190.4 \\
205.9\end{array}$ & $\begin{array}{r}8.95 \\
11.14 \\
12.62 \\
12.90 \\
12.81 \\
12.59 \\
12.10 \\
11.72\end{array}$ & $\begin{array}{r}9.48 \\
11.38 \\
12.86 \\
13.07 \\
12.97 \\
12.77 \\
12.46 \\
12.10\end{array}$ & $\begin{array}{r}9.94 \\
11.58 \\
12.97 \\
13.08 \\
12.98 \\
12.80 \\
12.60 \\
12.26\end{array}$ & $\begin{array}{l}10.30 \\
11.74 \\
12.96 \\
12.98 \\
12.89 \\
12.71 \\
12.62 \\
12.32\end{array}$ & $\begin{array}{l}10.56 \\
11.86 \\
12.86 \\
12.81 \\
12.74 \\
12.56 \\
12.57 \\
12.33\end{array}$ & $\begin{array}{l}10.76 \\
11.94 \\
12.70 \\
12.58 \\
12.54 \\
12.38 \\
12.48 \\
12.31\end{array}$ & $\begin{array}{l}10.89 \\
11.93 \\
12.49 \\
12.30 \\
12.28 \\
12.18 \\
12.33 \\
12.25\end{array}$ & $\begin{array}{l}11.00 \\
11.95 \\
12.98 \\
13.09 \\
12.99 \\
12.80 \\
12.62 \\
12.33\end{array}$ & $\begin{array}{c}\text { Degrees } \\
78 \\
67.5 \\
52.0 \\
48.1 \\
47.8 \\
48.4 \\
54.3 \\
59.3\end{array}$ & $\begin{array}{r}l b / i h p-h r \\
0.641 \\
.640 \\
.663 \\
.787 \\
.879 \\
.969 \\
1.049 \\
1.161\end{array}$ & $\begin{array}{r}{ }^{\circ} F \\
631 \\
703 \\
736 \\
725 \\
699 \\
669 \\
641 \\
610\end{array}$ \\
\hline & . & & & & 15 & 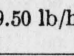 & $\pi$ & & & 47.8 & 0.639 & \\
\hline
\end{tabular}


TABLE 7.-Power and economy tests on blend 2

[Compression ratio 5.11]

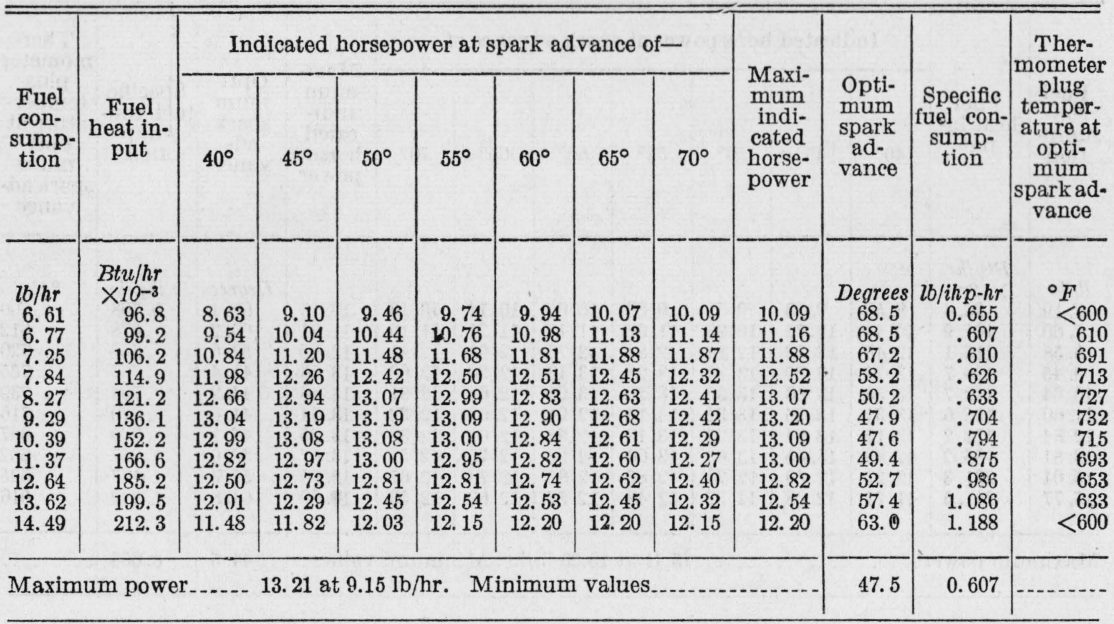

TABLE 8.-Power and economy tests on blend 3

[Compression ratio 5.11]

\begin{tabular}{|c|c|c|c|c|c|c|c|c|c|c|c|c|}
\hline \multirow[b]{2}{*}{$\begin{array}{l}\text { Fuel } \\
\text { con- } \\
\text { sump- } \\
\text { tion }\end{array}$} & \multirow[b]{2}{*}{$\begin{array}{c}\text { Fuel } \\
\text { heat in- } \\
\text { put }\end{array}$} & \multicolumn{7}{|c|}{ Indicated horsepower at spark advance of- } & \multirow{2}{*}{$\begin{array}{l}\text { Maxi- } \\
\text { mum } \\
\text { indi- } \\
\text { cated } \\
\text { horse- } \\
\text { power }\end{array}$} & \multirow[b]{2}{*}{$\begin{array}{l}\text { Opti- } \\
\text { mum } \\
\text { spark } \\
\text { ad- } \\
\text { vance }\end{array}$} & \multirow[b]{2}{*}{$\begin{array}{c}\text { Specific } \\
\text { fuel con- } \\
\text { sump- } \\
\text { tion }\end{array}$} & \multirow{2}{*}{$\begin{array}{l}\text { Ther- } \\
\text { mometer } \\
\text { plug } \\
\text { temper- } \\
\text { ature at } \\
\text { opti- } \\
\text { mum } \\
\text { spark ad- } \\
\text { vance }\end{array}$} \\
\hline & & $40^{\circ}$ & $45^{\circ}$ & $50^{\circ}$ & $55^{\circ}$ & $60^{\circ}$ & $65^{\circ}$ & $70^{\circ}$ & & & & \\
\hline $\begin{array}{r}l b / h r \\
6.37 \\
6.64 \\
7.18 \\
7.57 \\
8.08 \\
8.94 \\
9.83 \\
10.69 \\
11.72 \\
12.87 \\
13.88\end{array}$ & $\begin{array}{r}\text { Btu/hr } \\
\times 10^{-3} \\
94.7 \\
98.7 \\
106.7 \\
112.5 \\
120.1 \\
132.8 \\
146.1 \\
158.9 \\
174.2 \\
191.2 \\
206.3\end{array}$ & $\begin{array}{r}7.88 \\
9.30 \\
11.07 \\
12.06 \\
12.44 \\
12.84 \\
12.85 \\
12.68 \\
12.45 \\
11.98 \\
11.30\end{array}$ & $\begin{array}{r}8.56 \\
10.10 \\
11.41 \\
12.36 \\
12.69 \\
13.03 \\
13.04 \\
12.87 \\
12.70 \\
12.25 \\
11.70\end{array}$ & $\begin{array}{r}9.02 \\
10.53 \\
11.66 \\
12.49 \\
12.81 \\
13.12 \\
13.07 \\
12.93 \\
12.83 \\
12.46 \\
11.91\end{array}$ & $\begin{array}{r}9.33 \\
10.79 \\
11.79 \\
12.50 \\
12.83 \\
13.03 \\
12.98 \\
12.87 \\
12.77 \\
12.52 \\
11.98\end{array}$ & $\begin{array}{r}9.54 \\
10.95 \\
11.83 \\
12.42 \\
12.75 \\
12.85 \\
12.78 \\
12.72 \\
12.66 \\
12.50 \\
12.00\end{array}$ & $\begin{array}{r}9.65 \\
11.04 \\
11.82 \\
12.30 \\
12.60 \\
12.61 \\
12.53 \\
12.52 \\
12.46 \\
12.44 \\
11.97\end{array}$ & $\begin{array}{r}9.60 \\
11.04 \\
11.75 \\
12.13 \\
12.36 \\
12.33 \\
12.22 \\
12.24 \\
12.22 \\
12.35 \\
11.92\end{array}$ & $\begin{array}{r}9.66 \\
11.04 \\
11.83 \\
12.51 \\
12.83 \\
13.12 \\
13.08 \\
12.93 \\
12.83 \\
12.52 \\
12.00\end{array}$ & $\begin{array}{c}\text { Degrees } \\
66.4 \\
67.4 \\
60.5 \\
52.5 \\
53.2 \\
49.6 \\
49.0 \\
49.9 \\
51.6 \\
56.0 \\
58.6\end{array}$ & $\begin{array}{r}l b / i h p-h r \\
0.659 \\
.601 \\
.607 \\
.605 \\
.630 \\
.681 \\
.752 \\
.827 \\
.913 \\
1.028 \\
1.157\end{array}$ & $\begin{array}{r}{ }^{\circ} F \\
<600 \\
<600 \\
666 \\
717 \\
727 \\
732 \\
725 \\
696 \\
660 \\
627 \\
<600\end{array}$ \\
\hline & & & & & r. I & & & & & 49.0 & 0.600 & \\
\hline
\end{tabular}


TABLE 9.-Power and economy tests of 190-proof ethyl alcohol

[Compression ratio 9.91]

\begin{tabular}{|c|c|c|c|c|c|c|c|c|c|c|c|c|c|}
\hline \multirow[b]{2}{*}{$\begin{array}{l}\text { Fuel } \\
\text { con- } \\
\text { sump- } \\
\text { tion }\end{array}$} & \multirow[b]{2}{*}{$\begin{array}{c}\text { Fuel } \\
\text { heat } \\
\text { input }\end{array}$} & \multicolumn{8}{|c|}{ Indicated horsepower at spark advance of- } & \multirow[b]{2}{*}{$\begin{array}{c}\text { Maxi- } \\
\text { mum } \\
\text { indi- } \\
\text { cated } \\
\text { horse- } \\
\text { power }\end{array}$} & \multirow[b]{2}{*}{$\begin{array}{l}\text { Opti- } \\
\text { mum } \\
\text { spark } \\
\text { ad- } \\
\text { vance }\end{array}$} & \multirow[b]{2}{*}{$\begin{array}{c}\text { Specif- } \\
\text { ic } \\
\text { fuel } \\
\text { con- } \\
\text { sump- } \\
\text { tion }\end{array}$} & \multirow{2}{*}{$\begin{array}{l}\text { Ther- } \\
\text { mom- } \\
\text { eter } \\
\text { plug } \\
\text { tem- } \\
\text { pera- } \\
\text { ture } \\
\text { at } \\
\text { opti- } \\
\text { mum } \\
\text { spark } \\
\text { ad- } \\
\text { vance }\end{array}$} \\
\hline & & $25^{\circ}$ & $30^{\circ}$ & $35^{\circ}$ & $40^{\circ}$ & $45^{\circ}$ & $50^{\circ}$ & $55^{\circ}$ & $60^{\circ}$ & & & & \\
\hline $\begin{array}{r}l b / h r \\
8.64 \\
9.09 \\
9.71 \\
10.56 \\
11.37 \\
12.21 \\
13.14 \\
14.08 \\
15.03\end{array}$ & $\begin{array}{c}\text { Btu/hr } \\
\times 10-3 \\
101.6 \\
106.9 \\
114.2 \\
124.2 \\
133.7 \\
143.6 \\
154.5 \\
165.6 \\
176.8\end{array}$ & $\begin{array}{l}13.32 \\
13.98 \\
14.82 \\
15.12 \\
15.31 \\
15.15 \\
14.70 \\
14.37 \\
13.43\end{array}$ & $\begin{array}{l}13.88 \\
14.70 \\
15.29 \\
15.85 \\
15.83 \\
15.76 \\
15.42 \\
15.03 \\
14.28\end{array}$ & $\begin{array}{l}14.32 \\
15.12 \\
15.64 \\
16.04 \\
16.13 \\
16.07 \\
15.79 \\
15.40 \\
14.71\end{array}$ & $\begin{array}{l}14.52 \\
15.20 \\
15.74 \\
16.05 \\
16.17 \\
16.16 \\
15.94 \\
15.58 \\
14.93\end{array}$ & $\begin{array}{l}14.47 \\
14.99 \\
15.52 \\
15.90 \\
15.94 \\
16.06 \\
15.87 \\
15.63 \\
15.02\end{array}$ & $\begin{array}{l}14.26 \\
14.63 \\
15.10 \\
15.48 \\
15.59 \\
15.78 \\
15.69 \\
15.58 \\
15.01\end{array}$ & $\begin{array}{l}13.88 \\
14.16 \\
14.65 \\
14.97 \\
15.17 \\
15.36 \\
15.44 \\
15.47 \\
14.93\end{array}$ & $\begin{array}{l}13.13 \\
13.58 \\
14.18 \\
14.42 \\
14.70 \\
14.81 \\
15.12 \\
15.25 \\
14.79\end{array}$ & \begin{tabular}{|l|}
14.53 \\
15.22 \\
15.75 \\
16.06 \\
16.18 \\
16.16 \\
15.94 \\
15.63 \\
15.02
\end{tabular} & $\begin{array}{c}\text { De- } \\
\text { grees } \\
41.6 \\
38.7 \\
39.0 \\
37.7 \\
37.1 \\
39.8 \\
40.7 \\
44.9 \\
47.8\end{array}$ & \begin{tabular}{|c|} 
lb/ihp- \\
$h r$ \\
0.595 \\
.597 \\
.617 \\
.658 \\
.703 \\
.756 \\
.824 \\
.901 \\
1.001
\end{tabular} & $\begin{array}{l}{ }^{\circ} F \\
727 \\
766 \\
798 \\
799 \\
771 \\
751 \\
727 \\
711 \\
662\end{array}$ \\
\hline & Maxir & &. & 8 & 45 & • & aime & & & & 37.3 & 0.595 & \\
\hline
\end{tabular}

TABLE 10.-Power and economy tests of 200-proof ethyl alcohol

[Compression ratio 9.91]

\begin{tabular}{|c|c|c|c|c|c|c|c|c|c|c|c|c|c|}
\hline \multirow[b]{2}{*}{$\begin{array}{l}\text { Fuel } \\
\text { con- } \\
\text { sump- } \\
\text { tion }\end{array}$} & \multirow[b]{2}{*}{$\begin{array}{l}\text { Fuel } \\
\text { heat } \\
\text { input }\end{array}$} & \multicolumn{8}{|c|}{ Indicated horsepower at spark advance of- } & \multirow[b]{2}{*}{$\begin{array}{l}\text { Maxi- } \\
\text { mum } \\
\text { indi- } \\
\text { cated } \\
\text { horse- } \\
\text { power }\end{array}$} & \multirow[b]{2}{*}{$\begin{array}{c}\text { Opti- } \\
\text { mum } \\
\text { spark } \\
\text { ad- } \\
\text { vance }\end{array}$} & \multirow[b]{2}{*}{$\begin{array}{c}\text { Spe- } \\
\text { cific } \\
\text { fuel } \\
\text { con- } \\
\text { sump- } \\
\text { tion }\end{array}$} & \multirow{2}{*}{$\begin{array}{l}\text { Ther- } \\
\text { mom- } \\
\text { eter } \\
\text { plug } \\
\text { tem- } \\
\text { pera- } \\
\text { ture } \\
\text { at } \\
\text { opti- } \\
\text { mum } \\
\text { spark } \\
\text { ad- } \\
\text { vance }\end{array}$} \\
\hline & & $25^{\circ}$ & $30^{\circ}$ & $35^{\circ}$ & $40^{\circ}$ & $45^{\circ}$ & $50^{\circ}$ & $55^{\circ}$ & $60^{\circ}$ & & & & \\
\hline $\begin{array}{r}l b / h r \\
7.48 \\
7.90 \\
8.76 \\
9.73 \\
10.48 \\
11.68 \\
12.78 \\
13.79 \\
15.04 \\
15.80\end{array}$ & $\begin{array}{c}\text { Btu/hr } \\
\times 10^{-3} \\
95.1 \\
100.5 \\
111.4 \\
123.8 \\
133.3 \\
148.6 \\
162.6 \\
175.4 \\
191.3 \\
201.0\end{array}$ & $\begin{array}{l}11.44 \\
12.75 \\
14.82 \\
15.77 \\
15.91 \\
15.66 \\
15.16 \\
14.26 \\
13.69 \\
13.58\end{array}$ & $\begin{array}{l}12.37 \\
13.67 \\
15.21 \\
16.03 \\
16.11 \\
16.02 \\
15.55 \\
15.05 \\
14.67 \\
14.41\end{array}$ & $\begin{array}{l}12.96 \\
14.02 \\
15.31 \\
16.10 \\
16.17 \\
16.12 \\
15.83 \\
15.46 \\
14.99 \\
14.76\end{array}$ & $\begin{array}{l}13.24 \\
14.16 \\
15.26 \\
16.03 \\
16.09 \\
16.09 \\
15.92 \\
15.59 \\
15.13 \\
14.91\end{array}$ & $\begin{array}{l}13.35 \\
14.12 \\
15.03 \\
15.76 \\
15.83 \\
15.80 \\
15.81 \\
15.57 \\
15.18 \\
14.97\end{array}$ & $\begin{array}{l}13.29 \\
13.84 \\
14.61 \\
15.28 \\
15.30 \\
15.38 \\
15.55 \\
15.38 \\
15.14 \\
14.94\end{array}$ & $\begin{array}{l}12.99 \\
13.35 \\
14.01 \\
14.71 \\
14.67 \\
14.82 \\
15.16 \\
15.04 \\
15.02 \\
14.83\end{array}$ & $\begin{array}{l}12.50 \\
12.56 \\
13.31 \\
14.13 \\
14.00 \\
14.09 \\
14.64 \\
14.63 \\
14.79 \\
14.64\end{array}$ & $\begin{array}{l}13.35 \\
14.17 \\
15.31 \\
16.10 \\
16.17 \\
16.12 \\
15.92 \\
15.60 \\
15.18 \\
14.97\end{array}$ & $\begin{array}{c}\text { De- } \\
\text { grees } \\
46.0 \\
41.4 \\
35.7 \\
35.0 \\
34.6 \\
35.7 \\
39.9 \\
41.5 \\
44.8 \\
45.6\end{array}$ & $\begin{array}{c}l b / i h p- \\
h r \\
0.560 \\
.558 \\
.572 \\
.604 \\
.648 \\
.725 \\
.803 \\
.884 \\
.991 \\
1.055\end{array}$ & $\begin{array}{r}{ }^{\circ} F \\
702 \\
760 \\
785 \\
805 \\
800 \\
762 \\
708 \\
693 \\
648 \\
<600\end{array}$ \\
\hline & $a x$ & & & & & 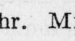 & & & & & 34.7 & 0.558 & \\
\hline
\end{tabular}


TABLE 11.-Power and economy tests of ether blend, fuel: $D$

[Compression ratio 8.60]

\begin{tabular}{|c|c|c|c|c|c|c|c|c|c|c|c|c|c|}
\hline \multirow[b]{2}{*}{$\begin{array}{l}\text { Fuel } \\
\text { con- } \\
\text { sump- } \\
\text { tion }\end{array}$} & \multirow[b]{2}{*}{$\begin{array}{l}\text { Fuel } \\
\text { heat } \\
\text { input }\end{array}$} & \multicolumn{8}{|c|}{ Indicated horsepower at spark advance of- } & \multirow[b]{2}{*}{$\begin{array}{c}\text { Maxi- } \\
\text { mum } \\
\text { indi- } \\
\text { cated } \\
\text { horse- } \\
\text { power }\end{array}$} & \multirow[b]{2}{*}{$\begin{array}{l}\text { Opti- } \\
\text { mum } \\
\text { spark } \\
\text { ad-- } \\
\text { vance }\end{array}$} & \multirow[b]{2}{*}{$\begin{array}{c}\text { Spe- } \\
\text { cific } \\
\text { fuel } \\
\text { con- } \\
\text { sump- } \\
\text { tion }\end{array}$} & \multirow{2}{*}{$\begin{array}{l}\text { Ther- } \\
\text { mom- } \\
\text { eter } \\
\text { plug } \\
\text { tem- } \\
\text { pera- } \\
\text { ture } \\
\text { at } \\
\text { opti- } \\
\text { mum } \\
\text { spark } \\
\text { ad- } \\
\text { vance }\end{array}$} \\
\hline & & $25^{\circ}$ & $30^{\circ}$ & $35^{\circ}$ & $40^{\circ}$ & $45^{\circ}$ & $50^{\circ}$ & $55^{\circ}$ & $60^{\circ}$ & & & & \\
\hline $\begin{array}{r}l b / h r \\
7.24 \\
7.92 \\
8.61 \\
9.21 \\
9.75 \\
10.43 \\
11.65 \\
12.68 \\
13.84 \\
14.21\end{array}$ & $\begin{array}{r}\text { Btu/hr } \\
\times 10^{-3} \\
97.1 \\
106.2 \\
115.5 \\
123.5 \\
130.7 \\
139.9 \\
156.2 \\
170.0 \\
185.6 \\
190.6\end{array}$ & $\begin{array}{l}11.38 \\
13.20 \\
14.34 \\
14.99 \\
15.03 \\
14.95 \\
14.53 \\
13.96 \\
13.35 \\
12.44\end{array}$ & $\begin{array}{l}12.25 \\
13.77 \\
14.99 \\
15.44 \\
15.48 \\
15.41 \\
15.10 \\
14.64 \\
13.96 \\
13.58\end{array}$ & $\begin{array}{l}12.93 \\
14.18 \\
15.18 \\
15.63 \\
15.68 \\
15.62 \\
15.41 \\
15.06 \\
14.52 \\
14.24\end{array}$ & $\begin{array}{l}13.25 \\
14.26 \\
15.17 \\
15.60 \\
15.65 \\
15.58 \\
15.47 \\
15.21 \\
14.84 \\
14.50\end{array}$ & $\begin{array}{l}13.28 \\
14.23 \\
14.92 \\
15.35 \\
15.37 \\
15.37 \\
15.34 \\
15.22 \\
14.90 \\
14.61\end{array}$ & $\begin{array}{l}13.12 \\
14.04 \\
14.56 \\
14.95 \\
14.99 \\
15.03 \\
15.09 \\
15.06 \\
14.87 \\
14.63\end{array}$ & $\begin{array}{l}12.82 \\
13.66 \\
14.07 \\
14.45 \\
14.49 \\
14.53 \\
14.73 \\
14.85 \\
14.76 \\
14.53\end{array}$ & $\begin{array}{l}12.36 \\
13.20 \\
13.46 \\
13.84 \\
13.90 \\
13.86 \\
14.23 \\
14.56 \\
14.53 \\
14.25\end{array}$ & $\begin{array}{l}13.30 \\
14.26 \\
15.20 \\
15.64 \\
15.70 \\
15.64 \\
15.48 \\
15.22 \\
14.90 \\
14.64\end{array}$ & $\begin{array}{c}\text { De- } \\
\text { grees } \\
43.4 \\
41.0 \\
36.5 \\
36.6 \\
36.7 \\
36.6 \\
39.0 \\
42.4 \\
45.6 \\
48.1\end{array}$ & $\begin{array}{c}l b / i h p- \\
h r \\
0.544 \\
.555 \\
.566 \\
.589 \\
.621 \\
.667 \\
.753 \\
.833 \\
.929 \\
.971\end{array}$ & $\begin{array}{c}{ }^{\circ} F \\
690 \\
765 \\
780 \\
798 \\
787 \\
773 \\
730 \\
697 \\
652 \\
640\end{array}$ \\
\hline & $\operatorname{tgan}$ & & & & & • & & & & & 36.5 & 0.545 & \\
\hline
\end{tabular}

TABLE 12.-Power and economy tests of blend 1

[Compression ratio 8.18]

\begin{tabular}{|c|c|c|c|c|c|c|c|c|c|c|c|c|c|}
\hline \multirow[b]{2}{*}{$\begin{array}{l}\text { Fuel } \\
\text { con- } \\
\text { sump- } \\
\text { tion }\end{array}$} & \multirow[b]{2}{*}{$\begin{array}{l}\text { Fuel } \\
\text { heat } \\
\text { input }\end{array}$} & \multicolumn{8}{|c|}{ Indicated horsepower at spark advance of- } & \multirow[b]{2}{*}{$\begin{array}{l}\text { Maxi- } \\
\text { mum } \\
\text { indi- } \\
\text { cated } \\
\text { horse- } \\
\text { power }\end{array}$} & \multirow[b]{2}{*}{$\begin{array}{c}\text { Opti- } \\
\text { mum } \\
\text { spark } \\
\text { ad- } \\
\text { vance }\end{array}$} & \multirow[b]{2}{*}{$\begin{array}{c}\text { Spe- } \\
\text { cific } \\
\text { fuel } \\
\text { con- } \\
\text { sump- } \\
\text { tion }\end{array}$} & \multirow{2}{*}{$\begin{array}{l}\text { Ther- } \\
\text { mom- } \\
\text { eter } \\
\text { plug } \\
\text { tem- } \\
\text { pera- } \\
\text { ture } \\
\text { at } \\
\text { opti- } \\
\text { mum } \\
\text { spark } \\
\text { ad- } \\
\text { vance }\end{array}$} \\
\hline & & $25^{\circ}$ & $30^{\circ}$ & $35^{\circ}$ & $40^{\circ}$ & $45^{\circ}$ & $50^{\circ}$ & $55^{\circ}$ & $60^{\circ}$ & & & & \\
\hline $\begin{array}{r}l b / h r \\
6.25 \\
6.61 \\
6.96 \\
7.48 \\
8.42 \\
9.44 \\
10.37 \\
11.52 \\
12.48 \\
13.39\end{array}$ & $\begin{array}{c}\text { Btu/hr } \\
\times 10-3 \\
89.9 \\
95.1 \\
100.1 \\
107.6 \\
121.1 \\
135.7 \\
149.1 \\
165.5 \\
179.5 \\
192.5\end{array}$ & $\begin{array}{r}8.57 \\
10.46 \\
11.78 \\
12.94 \\
14.34 \\
14.36 \\
14.16 \\
13.62 \\
12.92 \\
11.96\end{array}$ & $\begin{array}{r}9.82 \\
11.53 \\
12.93 \\
13.59 \\
14.74 \\
14.82 \\
14.74 \\
14.30 \\
13.73 \\
13.09\end{array}$ & $\begin{array}{l}10.72 \\
12.15 \\
13.32 \\
14.13 \\
14.98 \\
15.04 \\
14.98 \\
14.67 \\
14.23 \\
13.66\end{array}$ & $\begin{array}{l}11.32 \\
12.52 \\
13.47 \\
14.30 \\
15.04 \\
15.10 \\
15.04 \\
14.82 \\
14.47 \\
13.94\end{array}$ & $\begin{array}{l}11.73 \\
12.75 \\
13.47 \\
14.23 \\
14.87 \\
14.91 \\
14.96 \\
14.82 \\
14.58 \\
14.09\end{array}$ & $\begin{array}{l}12.00 \\
12.83 \\
13.30 \\
13.89 \\
14.49 \\
14.60 \\
14.67 \\
14.68 \\
14.59 \\
14.15\end{array}$ & $\begin{array}{l}12.12 \\
12.70 \\
12.99 \\
13.49 \\
14.05 \\
14.19 \\
14.27 \\
14.37 \\
14.46 \\
14.11\end{array}$ & $\begin{array}{l}12.04 \\
12.34 \\
12.61 \\
13.06 \\
13.58 \\
13.70 \\
13.82 \\
13.93 \\
14.16 \\
14.00\end{array}$ & $\begin{array}{l}12.12 \\
12.83 \\
13.48 \\
14.30 \\
15.05 \\
15.11 \\
15.04 \\
14.84 \\
14.60 \\
14.15\end{array}$ & 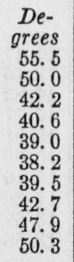 & $\begin{array}{c}l b / i h p- \\
h r \\
0.516 \\
.515 \\
.516 \\
.523 \\
.559 \\
.625 \\
.689 \\
.776 \\
.855 \\
.946\end{array}$ & $\begin{array}{l}{ }^{\circ} F \\
657 \\
696 \\
725 \\
778 \\
837 \\
771 \\
747 \\
711 \\
695 \\
654\end{array}$ \\
\hline & & 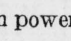 & *. & & & N & & & & & 38.2 & 0.514 & \\
\hline
\end{tabular}


TABLE 13.-Power and economy tests of blend 2

[Compression ratio 7.81]

\begin{tabular}{|c|c|c|c|c|c|c|c|c|c|c|c|c|c|}
\hline \multirow[b]{2}{*}{$\begin{array}{c}\text { Fuel } \\
\text { con- } \\
\text { sump- } \\
\text { tion }\end{array}$} & \multirow[b]{2}{*}{$\begin{array}{l}\text { Fuel } \\
\text { heat } \\
\text { input }\end{array}$} & \multicolumn{8}{|c|}{ Indicated horsepower at spark advance of- } & \multirow[b]{2}{*}{$\begin{array}{l}\text { Maxi- } \\
\text { mum } \\
\text { indi- } \\
\text { cated } \\
\text { horse } \\
\text { power }\end{array}$} & \multirow[b]{2}{*}{$\begin{array}{l}\text { Opti- } \\
\text { mum } \\
\text { spark } \\
\text { ad- } \\
\text { vance }\end{array}$} & \multirow[b]{2}{*}{$\begin{array}{c}\text { Spe- } \\
\text { cific } \\
\text { fuel } \\
\text { con- } \\
\text { sump- } \\
\text { tion }\end{array}$} & \multirow{2}{*}{$\begin{array}{l}\text { Ther- } \\
\text { mom- } \\
\text { eter } \\
\text { plug } \\
\text { tem- } \\
\text { pera- } \\
\text { ture } \\
\text { at } \\
\text { opti- } \\
\text { mum } \\
\text { spark } \\
\text { ad- } \\
\text { vance }\end{array}$} \\
\hline & & $25^{\circ}$ & $30^{\circ}$ & $35^{\circ}$ & $40^{\circ}$ & $45^{\circ}$ & $50^{\circ}$ & $55^{\circ}$ & $60^{\circ}$ & & & & \\
\hline $\begin{array}{r}l b / h r \\
6.98 \\
7.38 \\
8.28 \\
9.36 \\
9.98 \\
11.03 \\
12.03 \\
12.56\end{array}$ & $\begin{array}{c}\text { Btu/hr } \\
\times 10^{-3} \\
102.3 \\
108.1 \\
121.3 \\
137.1 \\
146.2 \\
161.6 \\
176.2 \\
184.0\end{array}$ & $\begin{array}{l}11.69 \\
13.10 \\
14.12 \\
14.16 \\
13.90 \\
13.59 \\
12.70 \\
12.20\end{array}$ & $\begin{array}{l}12.45 \\
13.55 \\
14.66 \\
14.66 \\
14.56 \\
14.24 \\
13.42 \\
12.92\end{array}$ & $\begin{array}{l}13.06 \\
13.97 \\
14.91 \\
15.02 \\
14.91 \\
14.58 \\
13.98 \\
13.56\end{array}$ & $\begin{array}{l}13.43 \\
14.27 \\
14.93 \\
15.04 \\
14.95 \\
14.70 \\
14.34 \\
14.03\end{array}$ & $\begin{array}{l}13.50 \\
14.11 \\
14.81 \\
14.88 \\
14.85 \\
14.72 \\
14.46 \\
14.18\end{array}$ & $\begin{array}{l}13.31 \\
13.82 \\
14.48 \\
14.66 \\
14.67 \\
14.62 \\
14.46 \\
14.21\end{array}$ & $\begin{array}{l}12.97 \\
13.48 \\
14.08 \\
14.32 \\
14.41 \\
14.41 \\
14.33 \\
14.13\end{array}$ & $\begin{array}{l}12.57 \\
13.10 \\
13.66 \\
13.83 \\
14.05 \\
14.07 \\
13.93 \\
13.93\end{array}$ & $\begin{array}{l}13.51 \\
14.27 \\
14.94 \\
15.05 \\
14.96 \\
14.72 \\
14.48 \\
14.21\end{array}$ & $\begin{array}{c}\text { De. } \\
\text { grees } \\
43.7 \\
40.6 \\
38.1 \\
38.1 \\
39.4 \\
42.9 \\
47.5 \\
48.9\end{array}$ & $\begin{array}{c}l b / i h p- \\
h r \\
0.517 \\
.517 \\
.554 \\
.622 \\
.668 \\
.749 \\
.831 \\
.884\end{array}$ & $\begin{array}{c}{ }^{\circ} F \\
737 \\
775 \\
780 \\
760 \\
742 \\
714 \\
689 \\
659\end{array}$ \\
\hline & xim & 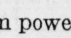 & 150 & 9.10 & ar. & $\operatorname{nim}$ & & & & & 37.8 & 0.514 & \\
\hline
\end{tabular}

TABLE 14.-Power and economy tests of blend 3

[Compression ratio 7.43]

\begin{tabular}{|c|c|c|c|c|c|c|c|c|c|c|c|c|c|}
\hline \multirow[b]{2}{*}{$\begin{array}{l}\text { Fuel } \\
\text { con- } \\
\text { sump- } \\
\text { tion }\end{array}$} & \multirow[b]{2}{*}{$\begin{array}{l}\text { Fuel } \\
\text { heat } \\
\text { input }\end{array}$} & \multicolumn{8}{|c|}{ Indicated horsepower at spark advance of- } & \multirow[b]{2}{*}{$\begin{array}{l}\text { Maxi- } \\
\text { mum } \\
\text { indi- } \\
\text { cated } \\
\text { horse } \\
\text { power }\end{array}$} & \multirow[b]{2}{*}{$\begin{array}{l}\text { Opti } \\
\text { mum } \\
\text { spark } \\
\text { ad- } \\
\text { vance }\end{array}$} & \multirow[b]{2}{*}{$\begin{array}{c}\text { Spe- } \\
\text { cific } \\
\text { fuel } \\
\text { con- } \\
\text { sump- } \\
\text { tion }\end{array}$} & \multirow{2}{*}{$\begin{array}{l}\text { Ther- } \\
\text { mom- } \\
\text { eter } \\
\text { pluy } \\
\text { terr } \\
\text { pera- } \\
\text { ture } \\
\text { at } \\
\text { opti- } \\
\text { mum } \\
\text { spark } \\
\text { ad- } \\
\text { vance }\end{array}$} \\
\hline & & $25^{\circ}$ & $30^{\circ}$ & $35^{\circ}$ & $40^{\circ}$ & $45^{\circ}$ & $50^{\circ}$ & $55^{\circ}$ & $60^{\circ}$ & & & & \\
\hline $\begin{array}{r}l b / h r \\
6.74 \\
7.30 \\
8.28 \\
9.04 \\
9.90 \\
11.05 \\
12.31 \\
13.30\end{array}$ & $\begin{array}{l}\text { Btu/hr } \\
\times 10^{-3} \\
100.2 \\
108.5 \\
123.0 \\
134.3 \\
147.1 \\
164.2 \\
182.9 \\
197.6\end{array}$ & $\begin{array}{l}10.81 \\
12.32 \\
13.82 \\
14.09 \\
13.97 \\
13.58 \\
12.92 \\
11.94\end{array}$ & $\begin{array}{l}11.43 \\
12.99 \\
14.31 \\
14.52 \\
14.46 \\
14.02 \\
13.64 \\
12.88\end{array}$ & $\begin{array}{l}12.00 \\
13.52 \\
14.68 \\
14.76 \\
14.69 \\
14.40 \\
13.97 \\
13.45\end{array}$ & $\begin{array}{l}12.48 \\
13.75 \\
14.73 \\
14.80 \\
14.73 \\
14.52 \\
14.15 \\
13.71\end{array}$ & $\begin{array}{l}12.70 \\
13.82 \\
14.61 \\
14.68 \\
14.61 \\
14.49 \\
14.20 \\
13.82\end{array}$ & $\begin{array}{l}12.77 \\
13.77 \\
14.34 \\
14.36 \\
14.27 \\
14.29 \\
14.15 \\
13.82\end{array}$ & $\begin{array}{l}12.75 \\
13.60 \\
13.94 \\
13.96 \\
13.86 \\
13.97 \\
13.97 \\
13.71\end{array}$ & $\begin{array}{l}12.59 \\
13.14 \\
13.45 \\
13.52 \\
13.40 \\
13.60 \\
13.63 \\
13.45\end{array}$ & $\begin{array}{l}12.77 \\
13.82 \\
14.74 \\
14.80 \\
14.73 \\
14.53 \\
14.20 \\
13.84\end{array}$ & $\begin{array}{c}\begin{array}{c}\text { De- } \\
\text { grees }\end{array} \\
51.2 \\
45.8 \\
39.4 \\
38.6 \\
38.7 \\
41.7 \\
45.1 \\
47.5\end{array}$ & $\begin{array}{c}l b / i h p- \\
h r \\
0.528 \\
.528 \\
.562 \\
.611 \\
.672 \\
.760 \\
.867 \\
.961\end{array}$ & $\begin{array}{l}{ }^{\circ} F \\
716 \\
769 \\
784 \\
770 \\
729 \\
683 \\
662 \\
625\end{array}$ \\
\hline & xim & por & 14.8 & 8.80 & hr. & inim & & & & & 38.6 & 0.525 & | \\
\hline
\end{tabular}


TABLE 15.-Power and economy tests of reference gasoline-second run

\begin{tabular}{|c|c|c|c|c|c|c|c|c|c|c|c|}
\hline \multirow[b]{2}{*}{$\begin{array}{l}\text { Fuel } \\
\text { con- } \\
\text { sump- } \\
\text { tion }\end{array}$} & \multirow[b]{2}{*}{$\begin{array}{l}\text { Fuel } \\
\text { heat } \\
\text { input }\end{array}$} & \multicolumn{6}{|c|}{ Indicated horsepower at spark advance of- } & \multirow{2}{*}{$\begin{array}{l}\text { Maxi- } \\
\text { mum } \\
\text { indi- } \\
\text { cated } \\
\text { horse- } \\
\text { power }\end{array}$} & \multirow[b]{2}{*}{$\begin{array}{l}\text { Opti- } \\
\text { mum } \\
\text { spark } \\
\text { ad- } \\
\text { vance }\end{array}$} & \multirow[b]{2}{*}{$\begin{array}{c}\text { Specific } \\
\text { fuel } \\
\text { con- } \\
\text { sump- } \\
\text { tion }\end{array}$} & \multirow{2}{*}{$\begin{array}{l}\text { Ther- } \\
\text { mometer } \\
\text { plug } \\
\text { temper- } \\
\text { ature at } \\
\text { opti- } \\
\text { mum } \\
\text { spark } \\
\text { advance }\end{array}$} \\
\hline & & $30^{\circ}$ & $40^{\circ}$ & $50^{\circ}$ & $60^{\circ}$ & $70^{\circ}$ & $80^{\circ}$ & & & & \\
\hline $\begin{array}{r}l b / h r \\
4.74 \\
4.95 \\
5.04 \\
5.33 \\
5.35 \\
5.63 \\
5.94 \\
6.26 \\
6.64 \\
7.24 \\
8.22 \\
9.19 \\
10.07 \\
11.09\end{array}$ & $\begin{array}{r}\text { Btu/hr } \\
\times 10^{-3} \\
95.6 \\
99.8 \\
101.7 \\
107.5 \\
107.9 \\
113.6 \\
119.8 \\
126.3 \\
133.9 \\
146.0 \\
165.8 \\
185.4 \\
203.1 \\
223.7\end{array}$ & $\begin{array}{r}11.53 \\
11.72 \\
11.63 \\
\end{array}$ & $\begin{array}{l}10.92 \\
11.72 \\
11.95 \\
12.22 \\
12.28 \\
12.25 \\
11.90 \\
11.46 \\
10.88\end{array}$ & $\begin{array}{r}8.40 \\
10.22 \\
10.69 \\
11.38 \\
11.40 \\
12.08 \\
12.30 \\
12.34 \\
12.29 \\
12.21 \\
12.16 \\
11.89 \\
11.27 \\
9.80\end{array}$ & $\begin{array}{r}8.97 \\
10.58 \\
10.98 \\
11.56 \\
11.55 \\
12.04 \\
12.18 \\
12.12 \\
12.03 \\
11.86 \\
11.92 \\
11.90 \\
11.66 \\
10.58\end{array}$ & $\begin{array}{r}9.22 \\
10.65 \\
11.01 \\
11.56 \\
11.53 \\
11.89 \\
11.89 \\
11.79 \\
11.34 \\
11.61 \\
11.57 \\
11.05\end{array}$ & $\begin{array}{r}9.30 \\
10.63 \\
10.92 \\
11.46 \\
11.39 \\
\\
\end{array}$ & $\begin{array}{r}9.30 \\
10.65 \\
11.02 \\
11.58 \\
11.56 \\
12.08 \\
12.30 \\
12.35 \\
12.32 \\
12.30 \\
12.16 \\
11.94 \\
11.66 \\
11.09\end{array}$ & $\begin{array}{c}\text { Degrees } \\
83 . \\
72.2 \\
66.8 \\
64.5 \\
63.0 \\
52.1 \\
51.0 \\
47.2 \\
45.5 \\
44.1 \\
49.5 \\
55.3 \\
61.4 \\
74.0\end{array}$ & $\begin{array}{r}l b / i h p-h r \\
0.510 \\
.465 \\
.457 \\
.460 \\
.463 \\
.466 \\
.483 \\
.507 \\
.539 \\
.589 \\
.676 \\
.770 \\
.864 \\
1.000\end{array}$ & $\begin{array}{r}{ }^{\circ} F \\
<600 \\
670 \\
678 \\
758 \\
- \\
780 \\
810 \\
806 \\
785 \\
755 \\
710 \\
666 \\
625 \\
<600\end{array}$ \\
\hline & & & & & $\mathrm{Mir}$ & & & & 45.0 & 0.458 & \\
\hline
\end{tabular}

Maximum indicated power and optimum spark advance were then plotted against fuel consumption, as was the derived function, indicated specific fuel consumption. Figures 1 to 14 show these curves. The first runs were made on a reference gasoline of 70 motor octane number, composed of standard reference fuels $\mathrm{C}-12$ and A-6. As the engine operators were not familiar with the test method, the readings were somewhat erratic and did not adequately cover the mixture-ratio range, as shown in figure 1. For this reason, the gasoline run was repeated some months later, and is shown in figure 14. In the interim several changes had been made in the test engine, including the installation of a new camshaft, and reference fuels C-12 and A-6 were no longer available. A blend of $\mathrm{C}-13$ and $\mathrm{M}-4$, of 70 motor octane number, was therefore used. The maximum power shown in figure 14 is consequently about $3 \frac{1}{2}$ percent below that shown in figure 1 . As the engine changes are believed to be responsible for this difference, the power and fuel-consumption values shown in figure 14 were corrected accordingly. 


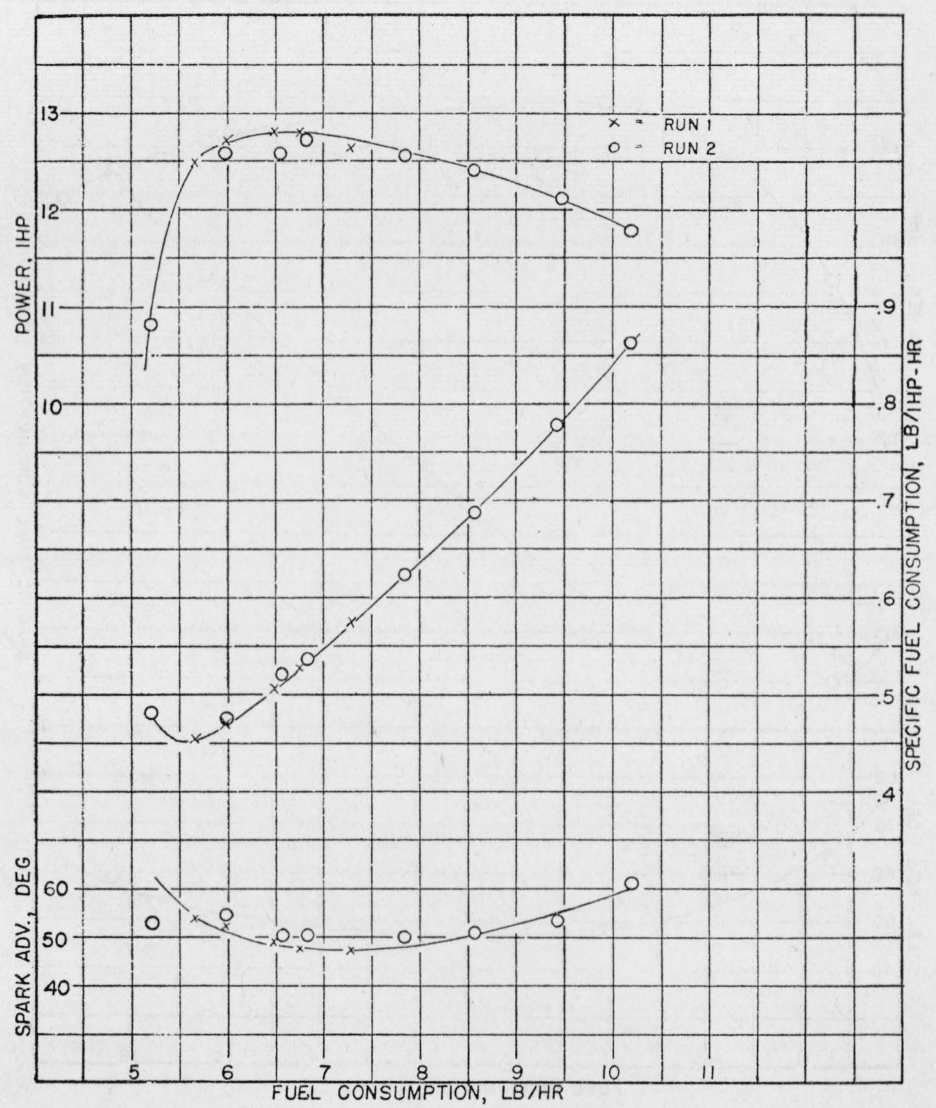

Figure 1.-Power, specific fuel consumption, and optimum spark advance for reference gasoline.

The engine was operated at a compression ratio of 5.11, which gave trace knock on this reference gasoline of 70 motor octane number. The two runs were made on successive days. 


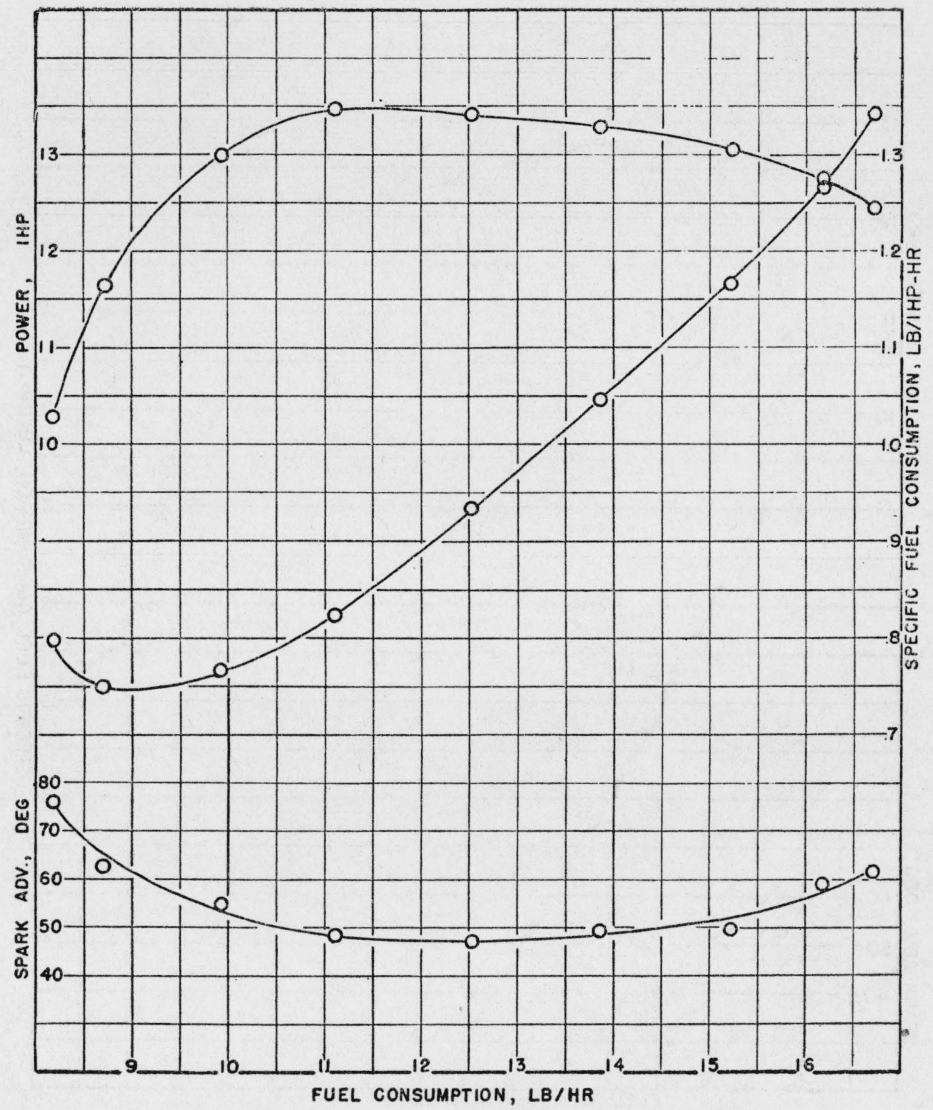

FIGURE 2.-Power, specific fuel consumption, and optimum spark advance for 190proof ethyl alcohol.

The engine was operated at a compression ratio of 5.11, which gave trace knock on the reference gasoline of 70 motor octane number. 


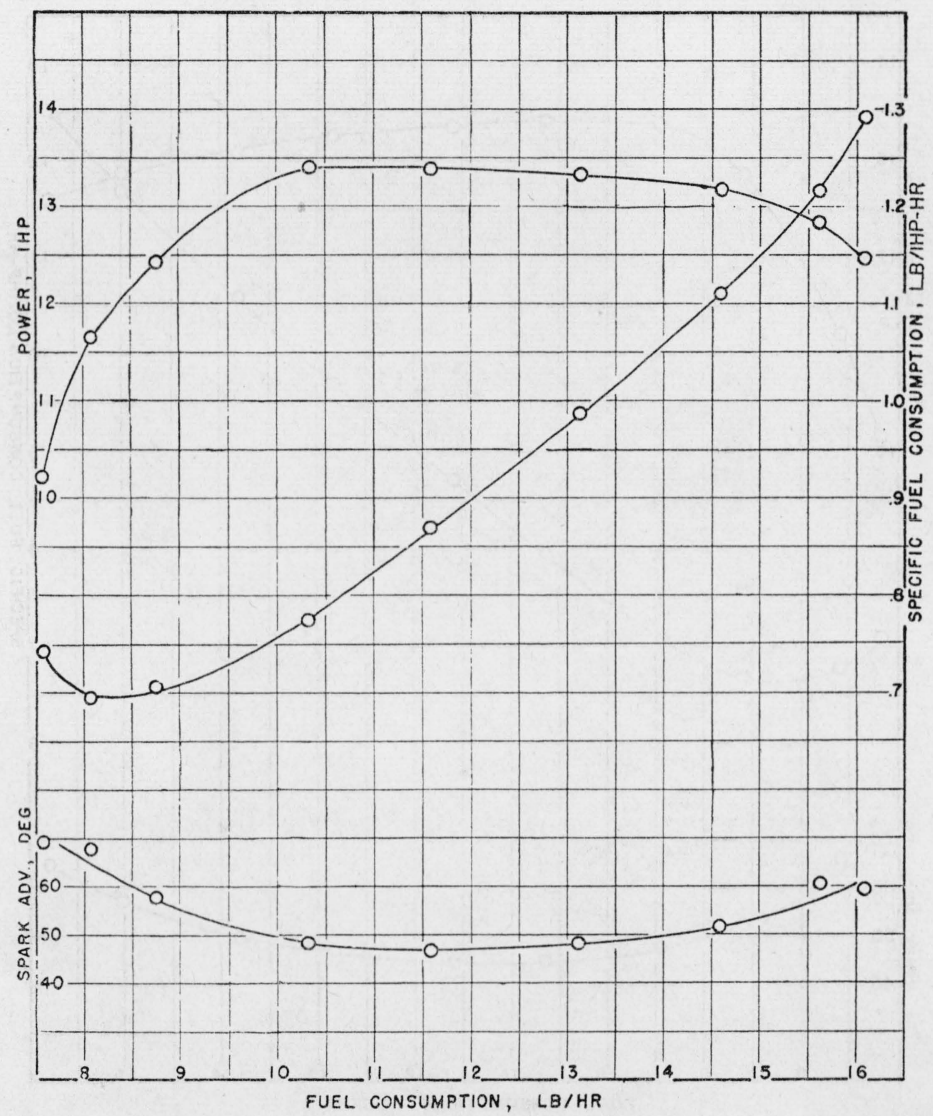

FIGURE 3.-Power, specific fuel consumption, and optimum spark advance for 200proof ethyl alcohol.

The engine was operated at a compression ratio of 5.11, which gave trace knock on the reference gasoline of 70 motor octane number. 


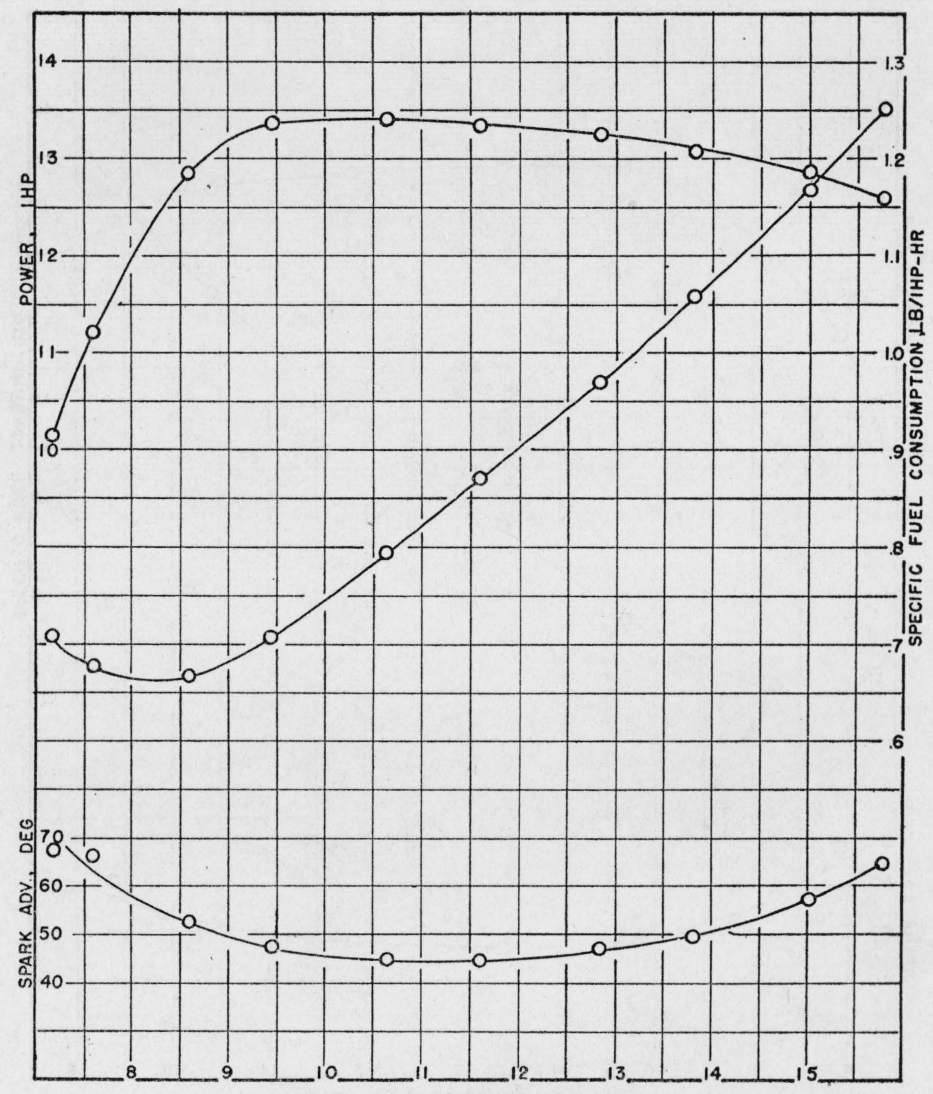

FUEL CONSUMPTION, LB/HR

FIgURE 4.-Power, specific fuel consumption, and optimum spark advance for a blend of 75 percent ethanol and 25 percent diethyl ether.

The engine was operated at a compression ratio of 5.11, which gave trace knock on the reference gasoline of 70 motor octane number 


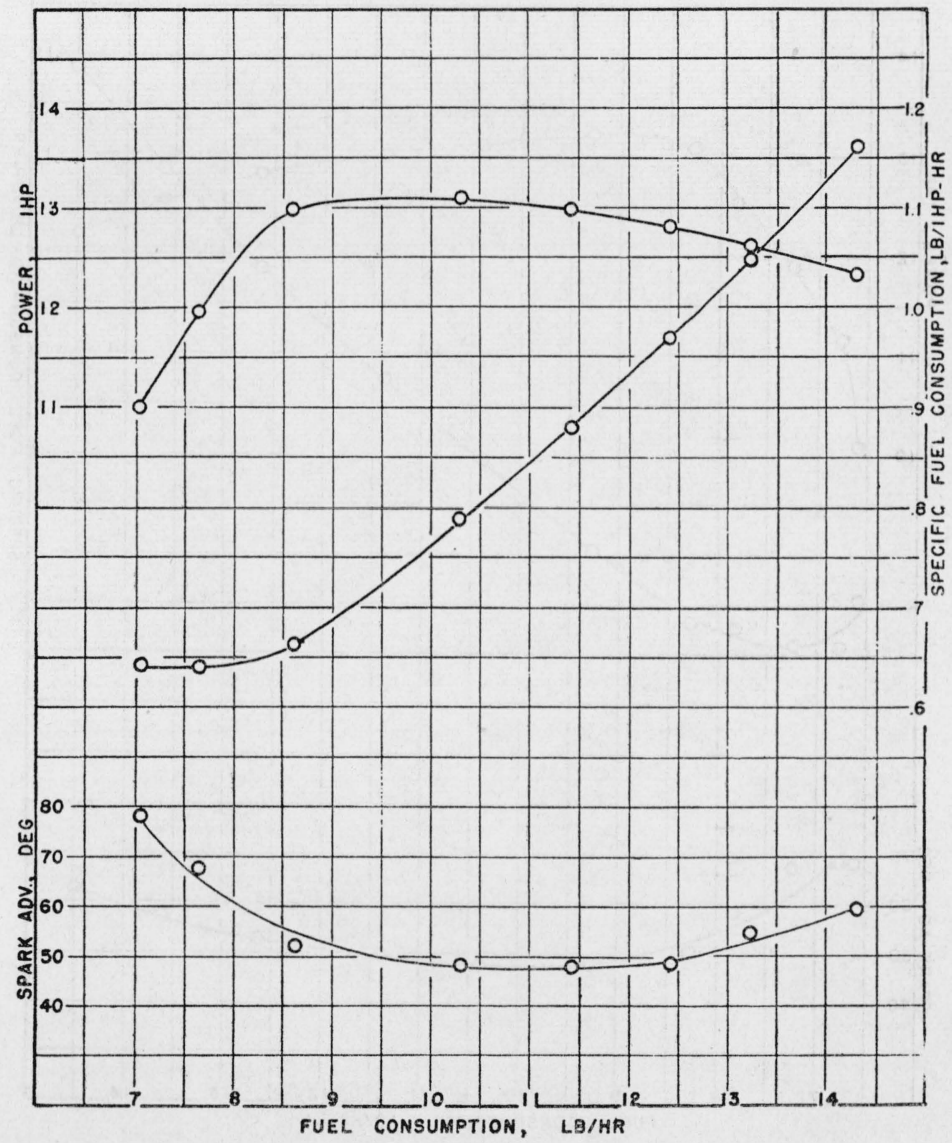

Figure 5.-Power, specific fuel consumption, and optimum spark advance for a blend of 50 percent acetone and 50 percent butanol.

The engine was operated at a compression ratio of 5.11, which gav $\theta$ trace knock on the reference gasoline of 70 motor octane number. 


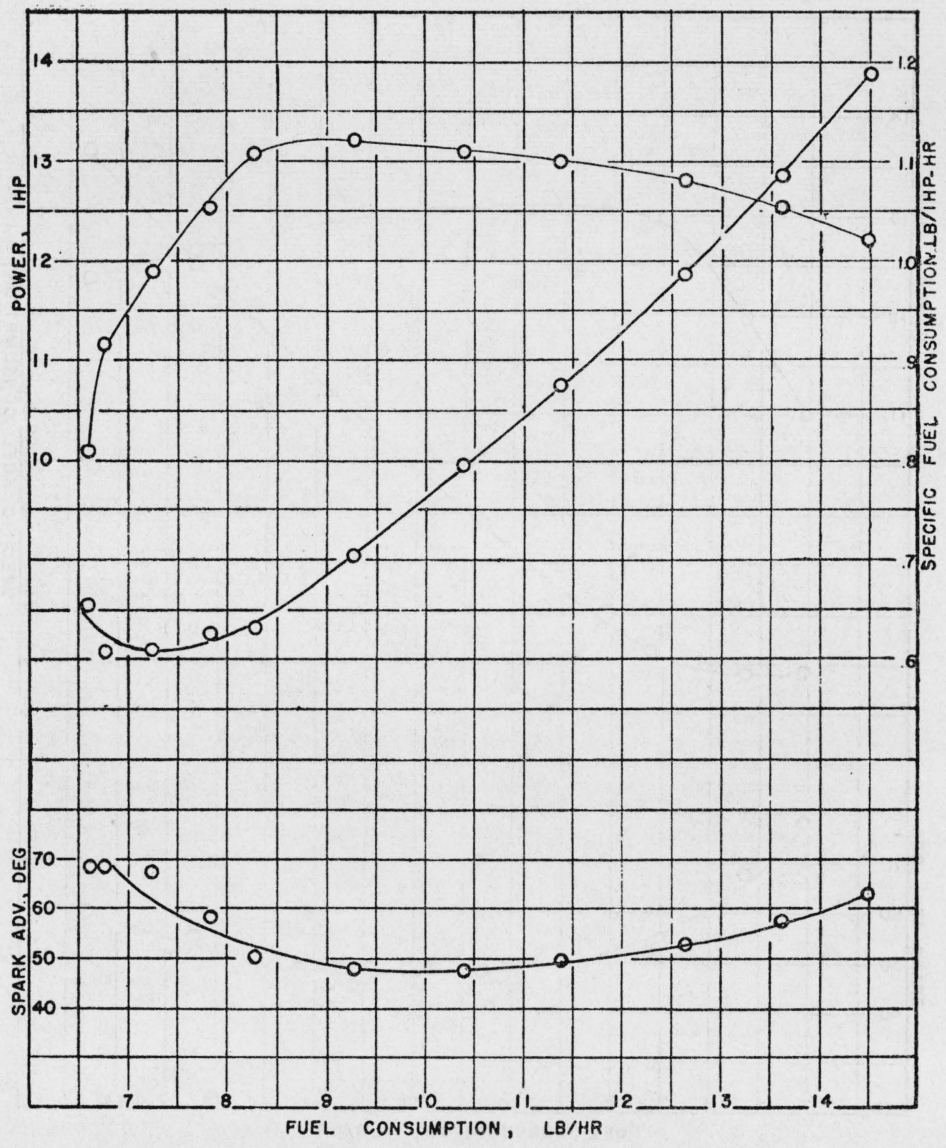

Figure 6.-Power, specific fuel consumption, and optimum spark advance for a blend of 27 percent acetone, 6 percent ethanol, and 67 percent butanol.

The engine was operated at a compression ratio of 5.11, which gave trace knock on the reference gasoline of 70 motor octane number. 


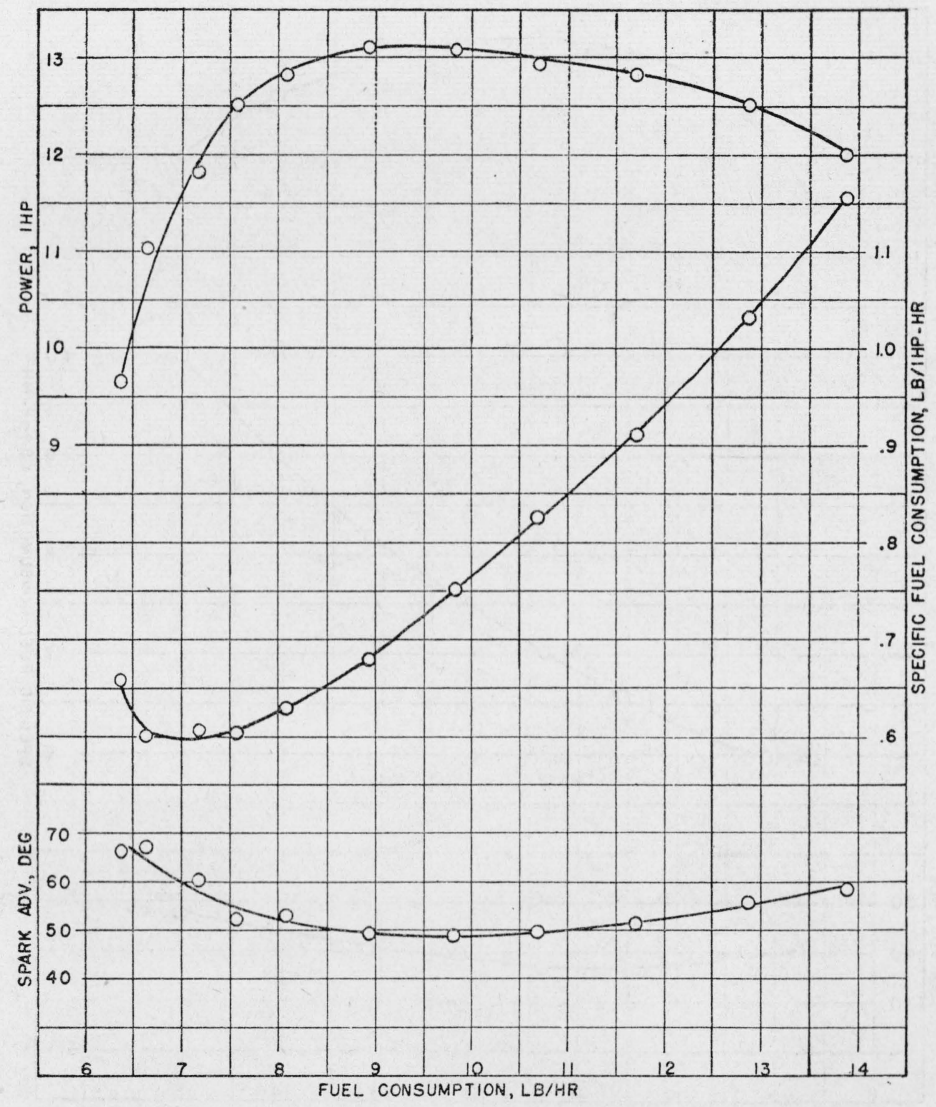

FIGURe 7.-Power, specific fuel consumption, and optimum spark advance for a blend of 28.5 percent acetone and 71.5 percent butanol.

The engine was operated at a compression ratio of 5.11, which gave trace knock on the reference gasoline of 70 motor octane number. 


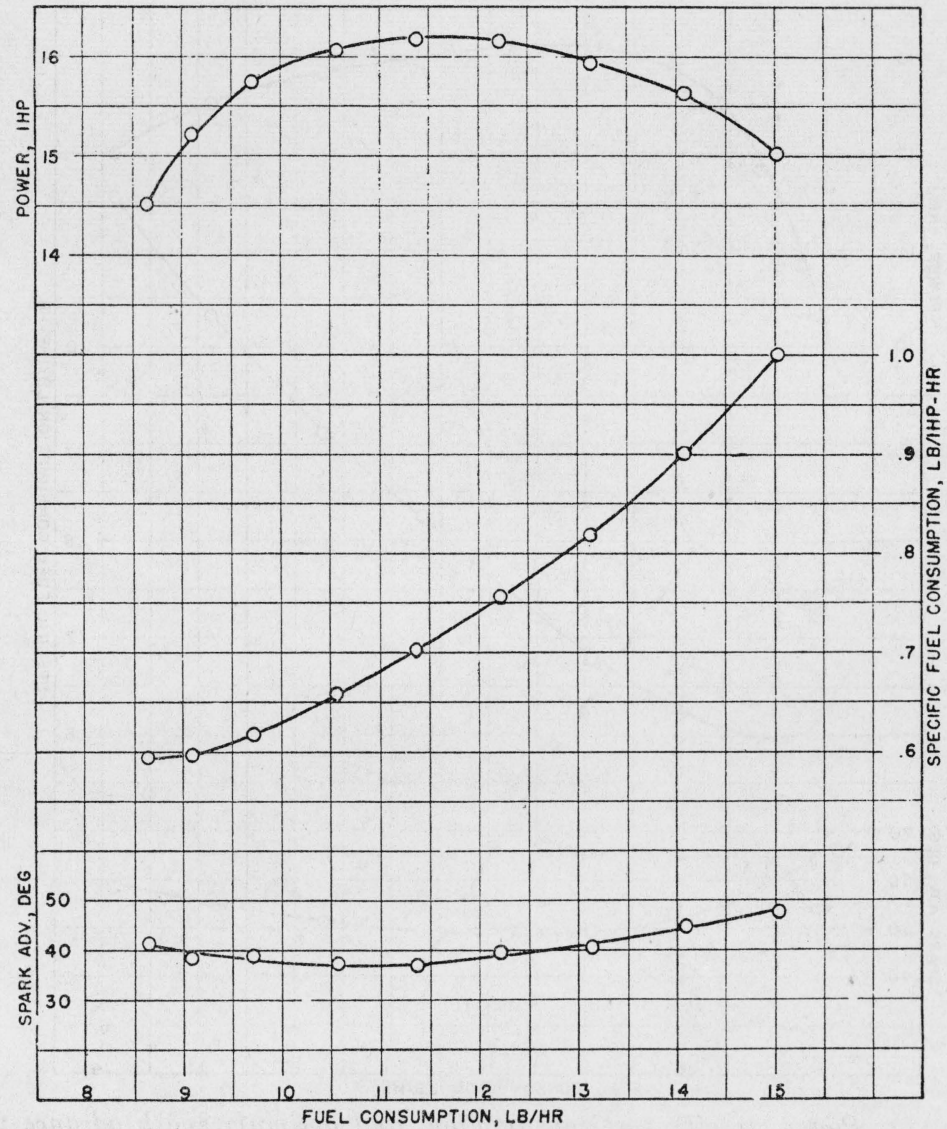

FIgure 8.-Power, specific fuel consumption, and optimum spark advance for 190proof ethyl alcohol.

The engine was operated at a compression ratio of 9.91, which gave trace knock on this fuel. 


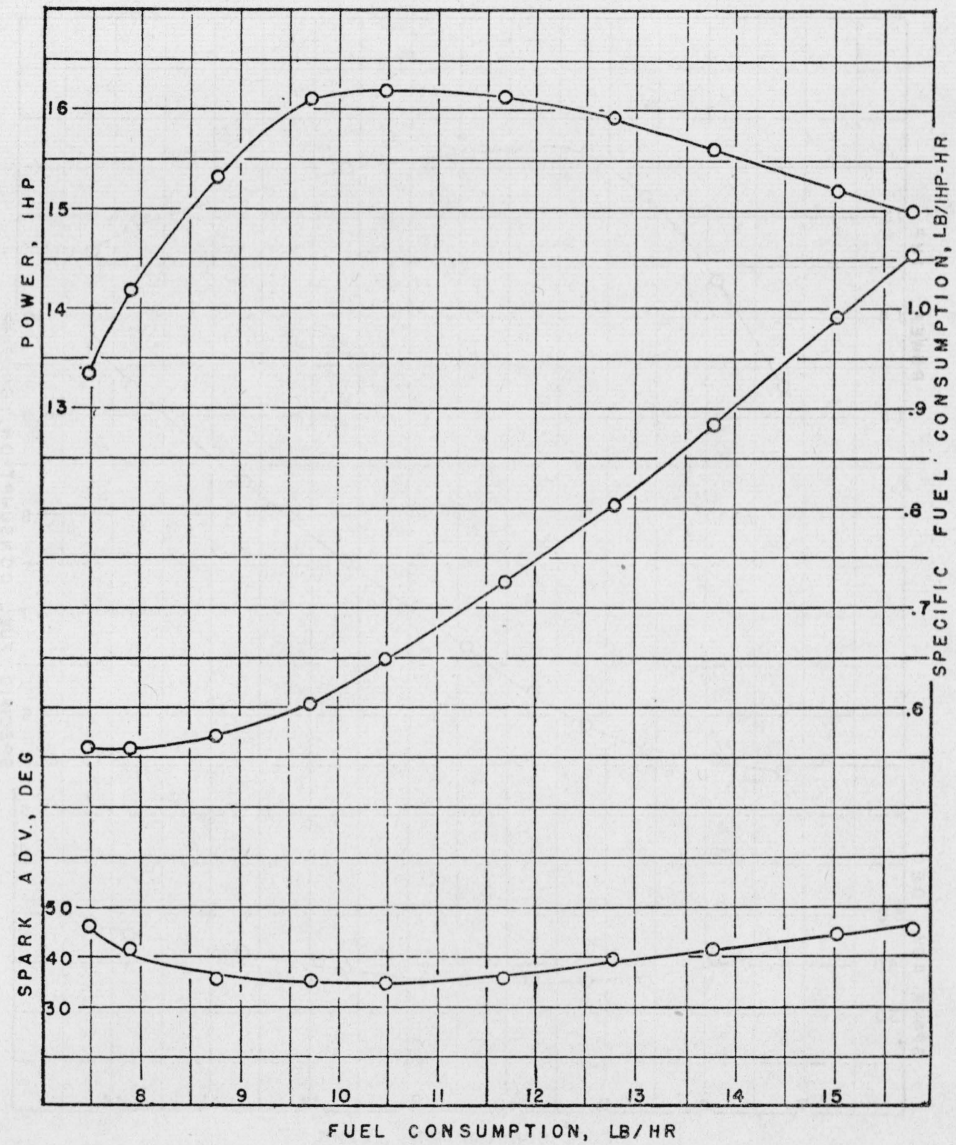

FIGUREI9.-Power, specific fuel consumption, and optimum spark advance for 200proof ethyl alcohol.

The engine was operated at a compression ratio of 9.91 , which gave trace knock on this fuel. 


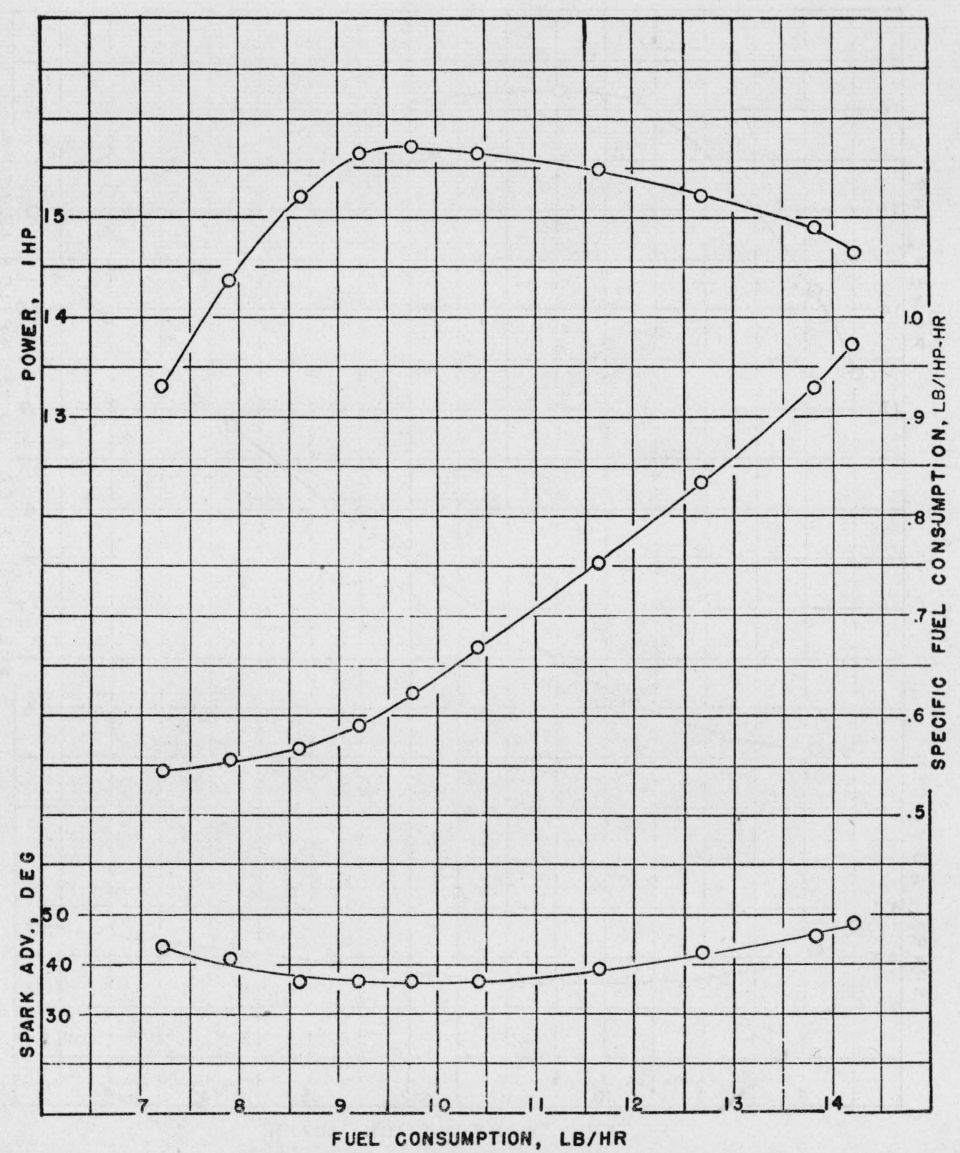

FIGURE 10.-Power, specific fuel consumption, and optimum spark advance for a blend of 75 percent ethanol and 25 percent diethyl other.

The engine was operated at a compression ratio of 8.60 , which gave trace knock on this fuel. 


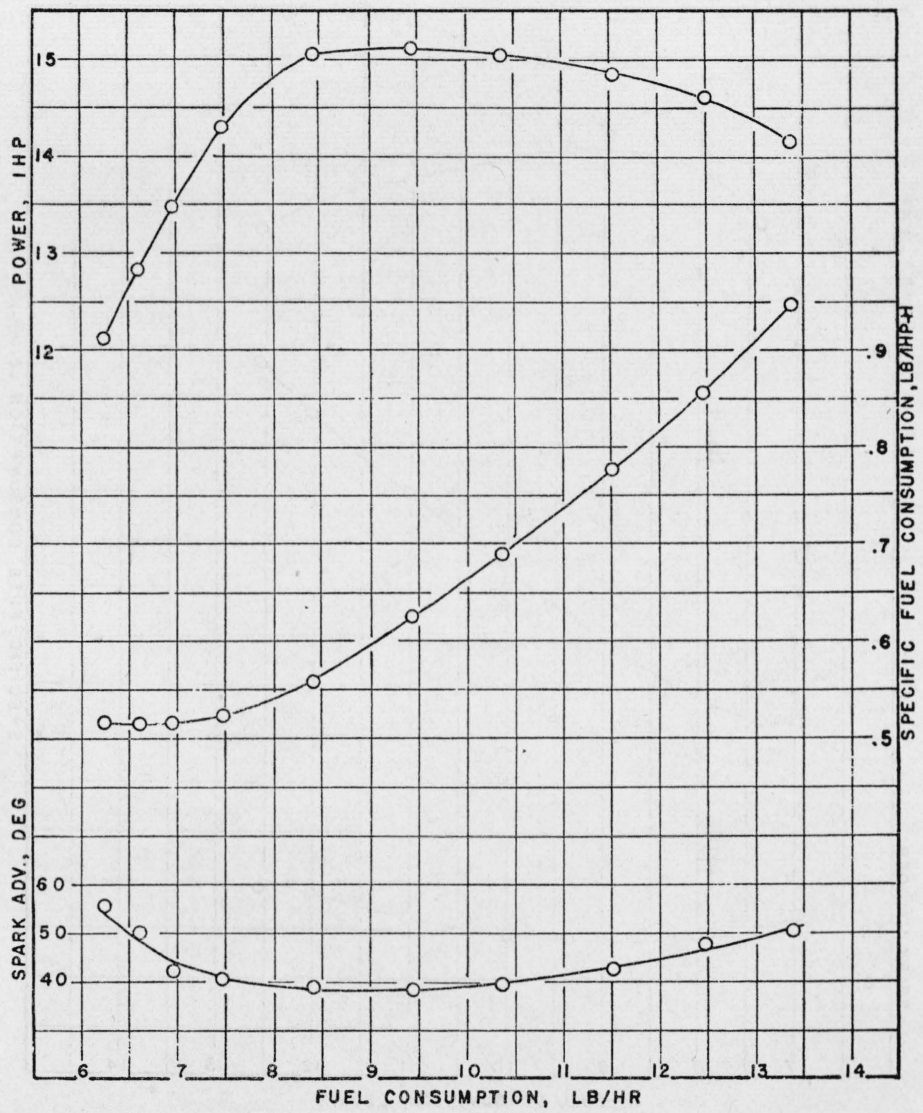

Figure 11.-Power, specific fuel consumption, and optimum spark advance for a blend of 50 percent acetone and 50 percent butanol.

The engine was operated at a compression ratio of 8.17 , which gave trace knock on this fuel. 


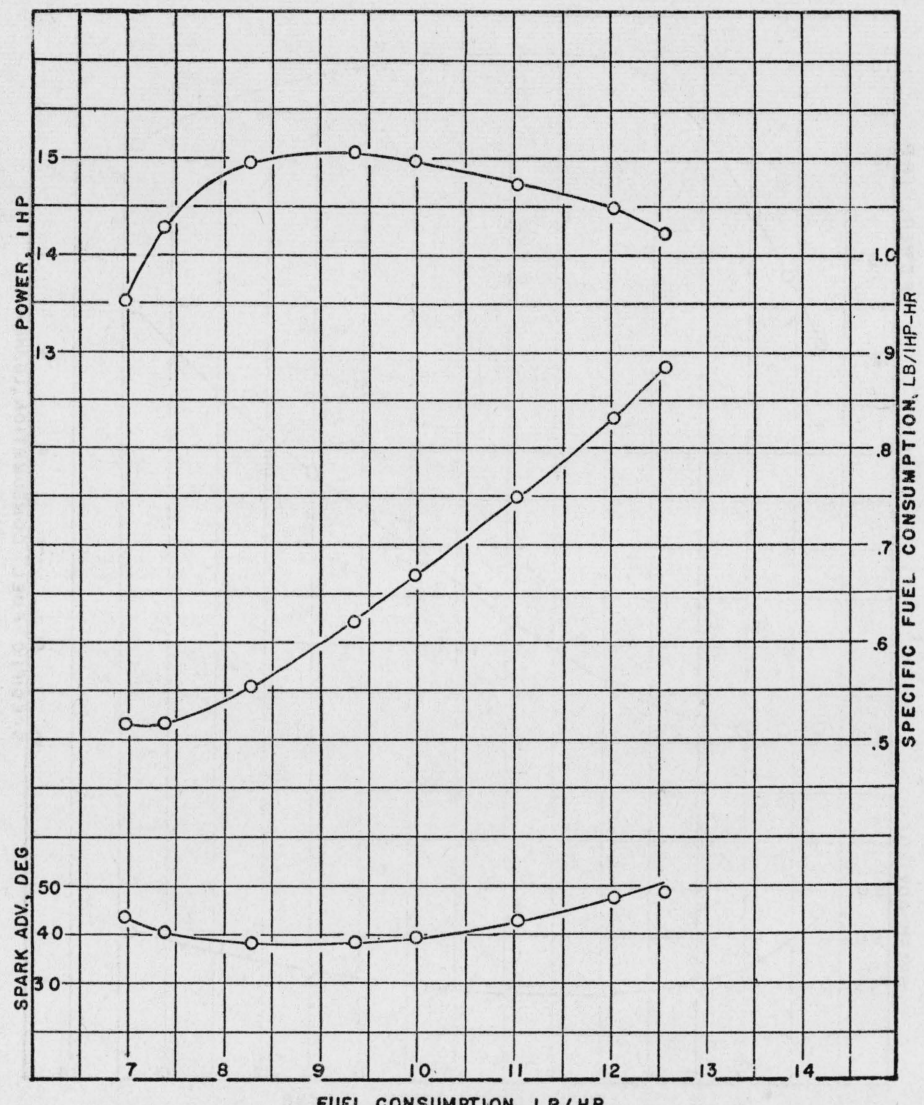

FiguRE 12.-Power, specific fuel consumption, and optimum spark advance for a blend of 27 percent acetone, 6 percent ethanol, and 67 percent butanol.

The engine was operated at a compression ratio of 7.80 , which gave trace knock on this fuel. 
Engine Tests of Substitute Fuels

23

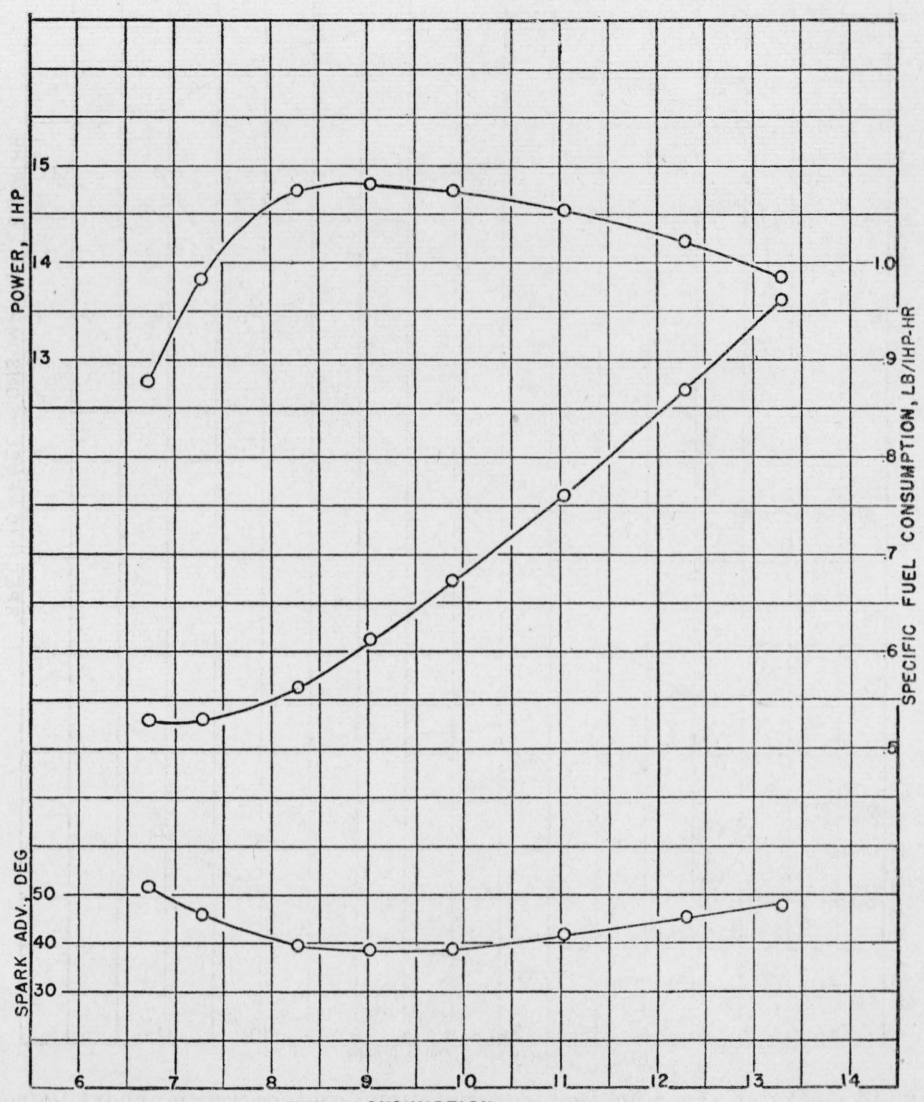

FUEL CONSUMPTION, LB/HR

FIGURE 13. -Power, specific fuel consumption, and optimum spark advance for a blend of 28.5 percent acetone and 71.5 percent butanol.

The engine was operated at a compression ratio of 7.43 , which gave trace knock on this fuel. 


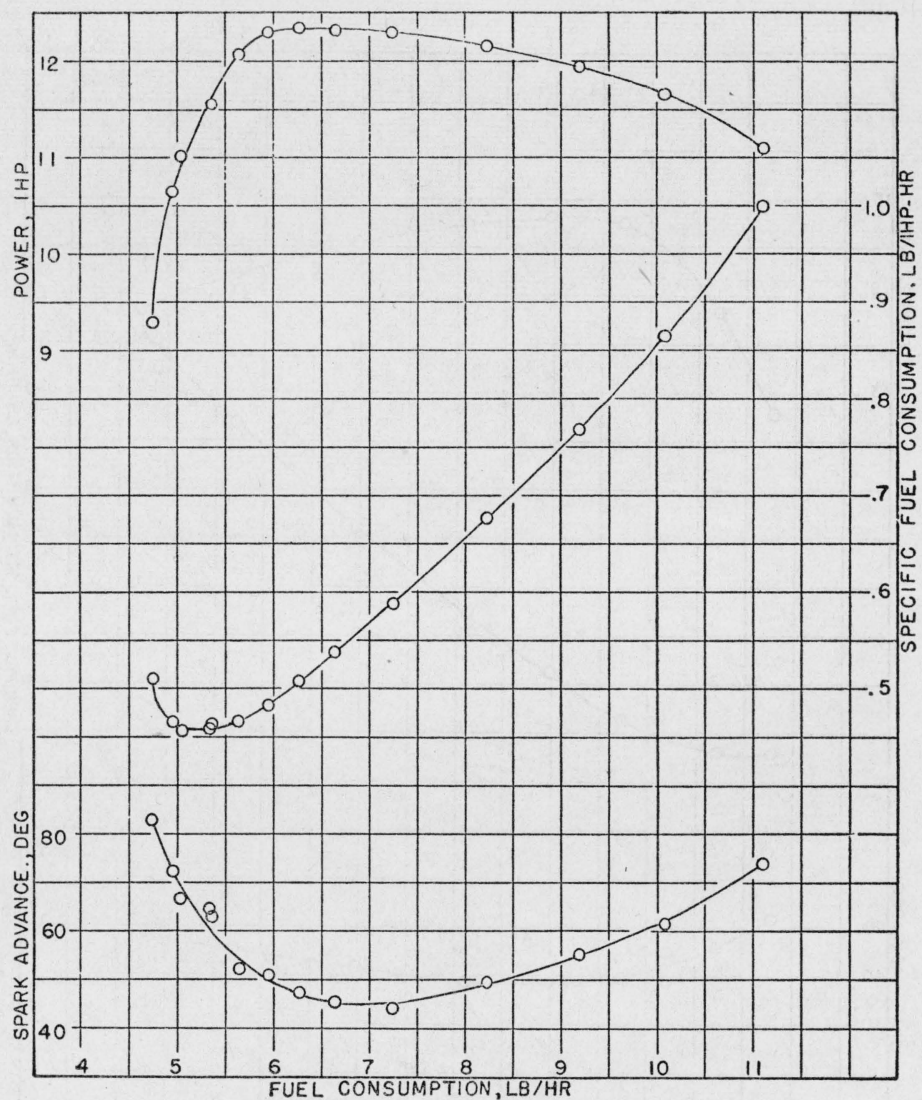

Figure 14.-Power, specific fuel consumption, and optimum spark advance for second reference gasoline.

The engine was operated at a compression ratio of 5.11, which gave trace knock on this reference gasoline of 70 motor octane number. This run, a repetition of that shown in figure 1, was made in order to cover more adequately the air-fuel range of interest. Several months elapsed between the test shown in figure 1 and this test, and interim engine changes altered the maximum power by about $3 \frac{1}{2}$ percent. The results were therefore corrected by an appropriate factor for all subsequent use. 


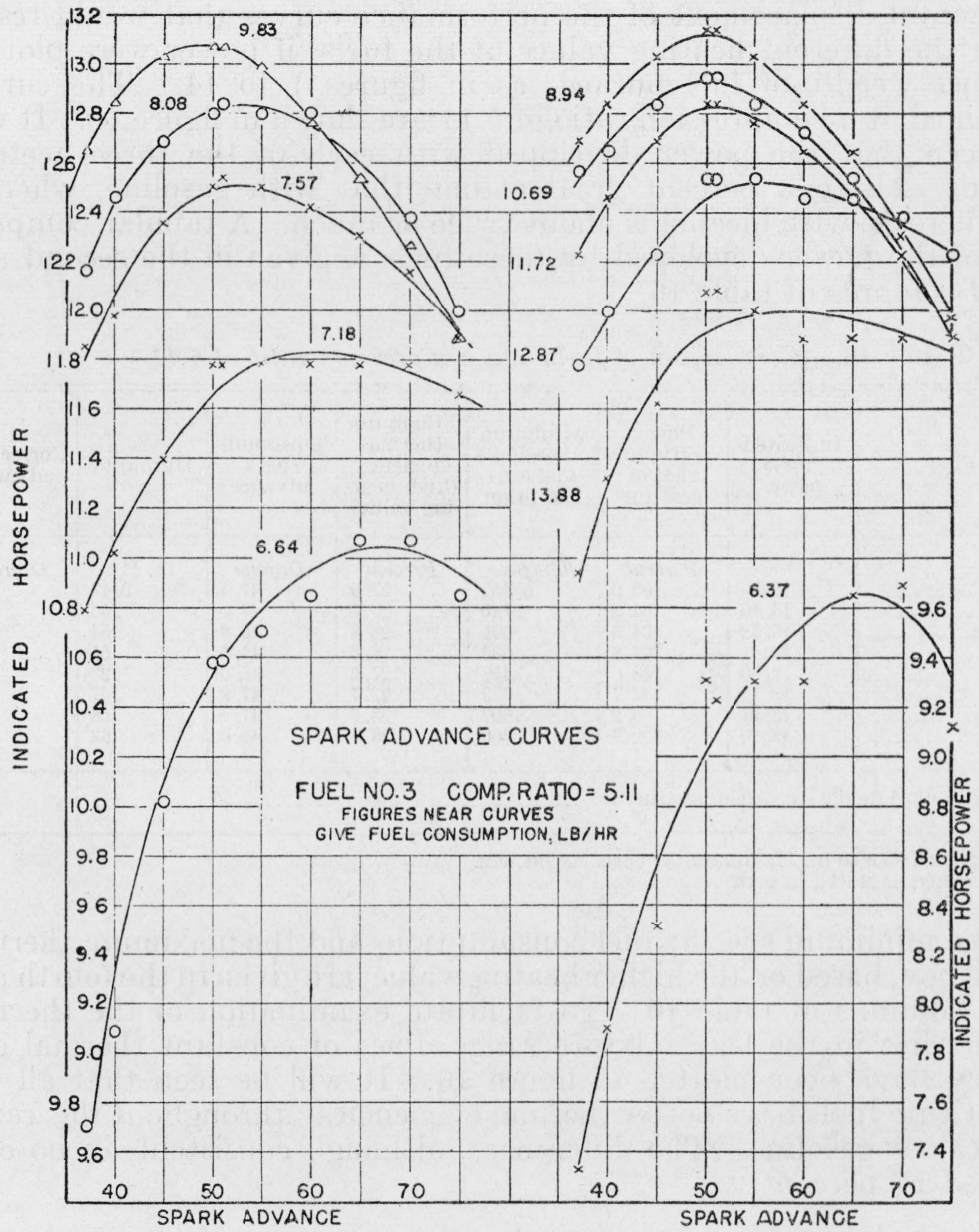

Figure 15.-Power versus spark advance at a series of fuel consumption rates.

These data, obtained at a compression ratio of 5.11, are typical for all of the fuels. The plotted points in figures 1 to 14 are the peaks of curves such as these. 


\section{DISCUSSION OF TEST RESULTS}

\section{POWER AT CONSTANT COMPRESSION RATIO}

For the purpose of facilitating comparison of performance over the mixture-ratio range, power was plotted against fuel heat input, calculated from the higher heating value ${ }^{1}$ of the fuel. This eliminates the horizontal displacement of the performance curves that would result from the different heating values of the fuels, if power were plotted against weight of fuel burned, as in figures 1 to 14 . The curves obtained at a compression ratio of 5.11 are shown in figure 16. It will be seen that the power developed with each of the three acetone blends is 2 to 3 percent greater than that with gasoline, whereas the increase with alcohol is about twice as much. A tabular comparison of the power developed by these fuels is given in the second and third columns of table 16 .

TABLE 16.-Comparison of fuels at a compression ratio of 5.11

\begin{tabular}{|c|c|c|c|c|c|c|c|}
\hline Fuel & $\begin{array}{c}\text { Indicated } \\
\text { horse- } \\
\text { power }\end{array}$ & $\begin{array}{l}\text { Power } \\
\text { ratio to } \\
\text { that of } \\
\text { gasoline }\end{array}$ & $\begin{array}{l}\text { Minimum } \\
\text { specific } \\
\text { fuel con- } \\
\text { sumption }\end{array}$ & $\begin{array}{l}\text { Maximum } \\
\text { thermal } \\
\text { efficiency } \\
\text { (high heat- } \\
\text { ing value) }\end{array}$ & $\begin{array}{l}\text { Optimum } \\
\text { spark } \\
\text { advance }\end{array}$ & Humidity & $\begin{array}{c}\text { Corrected }{ }^{1} \\
\text { advance }\end{array}$ \\
\hline $\begin{array}{l}\mathrm{A} \\
\mathrm{B} \\
\mathrm{B} \\
\mathrm{C} \\
\mathrm{D} \\
\mathrm{E} \\
\mathrm{E} \\
\mathrm{1} \\
2 \\
2\end{array}$ & $\begin{array}{l}12.80 \\
13.46 \\
13.38 \\
13.41 \\
12.80 \\
13.15 \\
13.21 \\
13.10\end{array}$ & $\begin{array}{c}\text { Percent } \\
100.0 \\
105.2 \\
104.5 \\
104.8 \\
100.0 \\
102.7 \\
103.2 \\
102.3\end{array}$ & $\begin{array}{r}l b / i h p-h r \\
0.452 \\
.746 \\
.695 \\
.663 \\
.458 \\
.639 \\
.607 \\
.600\end{array}$ & $\begin{array}{r}\text { Percent } \\
27.9 \\
29.0 \\
28.8 \\
28.6 \\
27.6 \\
27.7 \\
28.6 \\
28.5\end{array}$ & $\begin{array}{r}\text { Degrees } \\
47.5 \\
47.0 \\
46.9 \\
44.5 \\
45.0 \\
47.8 \\
47.5 \\
49.0\end{array}$ & $\begin{array}{c}\text { in. } \mathrm{Hg} \\
0.47 \\
.54 \\
.54 \\
.55 \\
.23 \\
.67 \\
.58 \\
.63\end{array}$ & $\begin{array}{r}\text { Degrees } \\
47.7 \\
46.7 \\
46.6 \\
44.1 \\
47.0 \\
46.6 \\
46.9 \\
48.0\end{array}$ \\
\hline \multicolumn{7}{|c|}{ Standard deviation (excluding fuel $D$ ) } & .5 \\
\hline
\end{tabular}

1 Corrected to $0.50 \mathrm{in}$. Hg humidity, basis $7.3^{\circ} / \mathrm{in}$. Hg.

2 Corrected as stated in text.

The minimum specific fuel consumption, and the maximum thermal efficiency, based on the higher heating value, are given in the fourth and fifth columns of table 16. To facilitate examination of the thermal efficiencies in the lean mixture range, lines of constant thermal efficiency have been plotted in figure 16 . It will be seen that all the substitute fuels have better thermal efficiencies ${ }^{2}$ throughout this range than does gasoline. The differences, although consistent, in no case exceed $1 \frac{1}{2}$ percent.

\section{COMPARISON OF POWER WITH CALCULATED VALUES}

Table 17 gives the calculated properties of theoretical mixtures of each of the fuels. The second column gives the calculated air-fuel ratio, and the third column gives the fuel heat per pound of dry air. The fourth column gives the relative heat per unit volume of charge at standard temperature and pressure, based on gasoline as unity. It can been seen from this column that the heating values of these fuel-air mixtures are essentially equal. The next four columns are steps in the computation of the potential cooling of the charge by fuel vaporiza-

\footnotetext{
1 See Appendix for values based on lower heating value.

2 It should be understood that a conventionalized calculation such as thermal efficiency does not accu rately describe the thermodynamic processes actually occurring in the engine.
} 


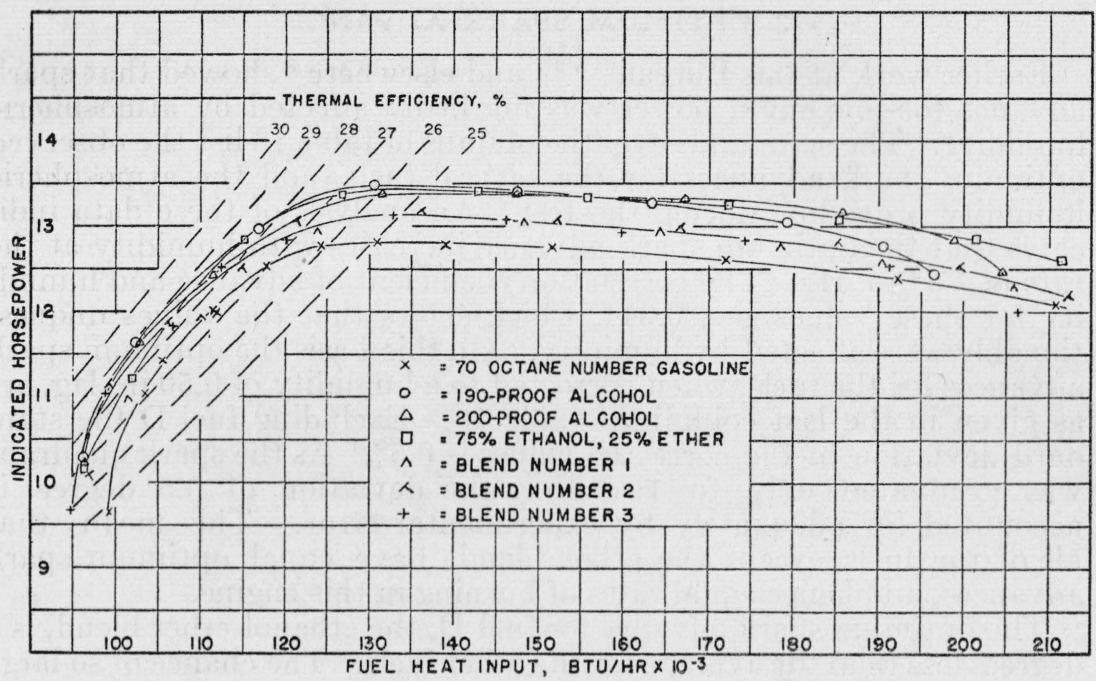

FigURe 16.-Power and thermal efficiency of the test fuels at a compression ratio of 5.11 .

When fuel consumption of dissimilar fuels is expressed in terms of fuel heat input, comparison of the power curves is facilitated. This also permits superposition of a scale of thermal efficiency.

tion. From this is found the volume of air that would be inducted if all of the fuel were vaporized, in terms of the gasoline-air mixture as unity. For comparison, the observed maximum power at equal compression ratio is tabulated in the last column, as the power obtainable from an engine has been shown ${ }^{3}$ to be determined by the air consumption of the engine. The agreement between the values in the last two columns is apparently a measure of the relative fuel vaporization at the moment of closure of the intake valve, as subsequent fuel vaporization would not affect air induction. Thus fuels 1 and 2 are nearer complete vaporization than is gasoline; fuel 3 and fuel $D$ are about the same; and the alcohols, fuels B and C, are less so, presumably because of the large potential cooling involved in their vaporization.

TABLE 17.-Properties of theoretical fuel-air mixtures

\begin{tabular}{|c|c|c|c|c|c|c|c|c|c|c|}
\hline Fuel & $\begin{array}{c}\text { Theoret- } \\
\text { ical air- } \\
\text { fuel ra- } \\
\text { tio }\end{array}$ & $\begin{array}{l}\text { Btu per } \\
\text { pound of } \\
\text { air at } \\
\text { theoret- } \\
\text { ical air- } \\
\text { fuel ratio }\end{array}$ & $\begin{array}{l}\text { Relative } \\
\text { heat per } \\
\text { unit vol- } \\
\text { ume of } \\
\text { charge }\end{array}$ & $\begin{array}{l}\text { Air (per- } \\
\text { centage } \\
\text { of vol- } \\
\text { ume of } \\
\text { charge) }\end{array}$ & $\begin{array}{c}\text { Latent } \\
\text { heat of } \\
\text { fuel va- } \\
\text { poriza- } \\
\text { tion a }\end{array}$ & $\begin{array}{c}\text { Specific } \\
\text { heat of } \\
\text { fuel }\end{array}$ & $\begin{array}{l}\text { Specific } \\
\text { heat of } \\
\text { charge }\end{array}$ & $\begin{array}{l}\text { Poten- } \\
\text { tial ceol- } \\
\text { ing of } \\
\text { charge }\end{array}$ & $\begin{array}{l}\text { Air (cal- } \\
\text { culated } \\
\text { percent- } \\
\text { age of } \\
\text { volume } \\
\text { of } \\
\text { charge) }\end{array}$ & $\begin{array}{l}\text { Ob- } \\
\text { served }\end{array}$ \\
\hline $\begin{array}{l}\mathrm{B} \\
\mathrm{C} \\
\mathrm{D} \\
\mathrm{E} \\
1\end{array}$ & $\begin{array}{r}8.19 \\
9.00 \\
9.51 \\
15.09 \\
10.36 \\
10.61 \\
10.72\end{array}$ & $\begin{array}{l}1,436 \\
1,413 \\
1,410 \\
1,337 \\
1,388 \\
1,380 \\
1,386\end{array}$ & $\begin{array}{r}\text { Percent } \\
100.2 \\
100.3 \\
101.0 \\
100.0 \\
101.1 \\
100.7 \\
101.4\end{array}$ & \begin{tabular}{|r|} 
Percent \\
92.0 \\
93.5 \\
94.3 \\
98.5 \\
95.9 \\
96.1 \\
96.2
\end{tabular} & $\begin{array}{r}\text { cal/g } \\
238 \\
204 \\
176 \\
75 \\
127 \\
133 \\
128\end{array}$ & $\begin{array}{c}\text { cal/g } \\
0.41 \\
.41 \\
.42 \\
.61 \\
.38 \\
.40 \\
.40\end{array}$ & $\begin{array}{c}\text { cal/g of } \\
\text { fuel } \\
2.37 \\
2.56 \\
2.69 \\
4.22 \\
2.86 \\
2.93 \\
2.96\end{array}$ & $\begin{array}{r}{ }^{\circ} C \\
100 \\
80 \\
65 \\
18 \\
44 \\
45 \\
43\end{array}$ & $\begin{array}{r}\text { Percent } \\
110.8 \\
107.4 \\
104.8 \\
100.0 \\
102.2 \\
102.6 \\
102.2\end{array}$ & $\begin{array}{r}\text { Percent } \\
105.2 \\
104.5 \\
104.8 \\
100.0 \\
102.7 \\
103.2 \\
102.3\end{array}$ \\
\hline
\end{tabular}

s From International Critical Tables.

Nat. Advisory Comm. Rep. No. 426 (1932). 


\section{OPTIMUM SPARK ADVANCE}

Earlier work at this Bureau ${ }^{4567}$ and elsewhere ${ }^{8}$ showed that spark advance for maximum power was markedly affected by atmospheric humidity. The sixth and seventh columns of table 16 list the observed optimum spark advances for the several fuels, and the atmospheric humidity prevailing during the test. An analysis of these data indicates that the optimum spark advance increases with humidity at the rate of $7.3^{\circ} \mathrm{in}$. Hg. The correlation coefficient of advance and humidity for these values is +0.944 , which shows that the values unquestionably were affected by humidity. On this basis the optimum spark advances for the fuels, when corrected to a humidity of $0.50 \mathrm{in}$. Hg, are as given in the last column of table 16. Excluding fuel D the standard deviation of the corrected values is $0.5^{\circ}$. As the spark quadrant was graduated only to 1 degree, the deviation of 0.5 degree is accounted for adequately by experimental error. This means that all of the fuels, except the ether blend, have equal optimum spark advances, and hence equal rates of burning in this engine.

The optimum spark advance for fuel D, the ethanol-ether blend, is 3 degrees less than the average for the other fuels. The chance of so large a deviation occurring through the operation of the factors that caused the experimental error for the other fuels is exceedingly small. It therefore appears that the ether blend actually burns some 7 percent faster than the other fuels.

\section{POWER AT COMPRESSION RATIO FOR TRACE KNOCK}

In figure 17 are plotted the runs in which each fuel was tested at the compression ratio for trace knock (for best-power mixture and optimum spark advance) and tabular values are given in the third to sixth columns of table 18. As in figure 16, lines of constant thermal efficiency are shown in figure 17. At the same heat input, a considerable increase in power and in thermal efficiency is shown by the substitute fuels, chiefly because of the permissible increase in compression ratio. The increase in power is approximately 83 percent of that calculated on an air-cycle basis, from the change of compression ratio. The apparent reason for the departure from air cycle is a decrease in the volumetric efficiency of the test engine with increase in compression ratio, the magnitude of the decrease being sufficient to account for the observed departure.

TABLE 18.-Comparison of fuels at compression ratio giving trace knock

\begin{tabular}{|c|c|c|c|c|c|c|c|c|}
\hline Fuel & $\begin{array}{l}\text { Compres- } \\
\text { sion ratio }\end{array}$ & $\begin{array}{c}\text { Indicated } \\
\text { horse } \\
\text { power }\end{array}$ & $\begin{array}{l}\text { Power ratio } \\
\text { to that of } \\
\text { gasoline }\end{array}$ & $\begin{array}{l}\text { Minimum } \\
\text { specific fuel } \\
\text { consump- } \\
\text { tion }\end{array}$ & $\begin{array}{c}\text { Maximum } \\
\text { thermal } \\
\text { efficiency }\end{array}$ & $\begin{array}{l}\text { Optimum } \\
\text { spark } \\
\text { advance }\end{array}$ & Humidity & $\begin{array}{l}\text { Corrected } \\
\text { advance }{ }^{1}\end{array}$ \\
\hline $\begin{array}{l}\mathrm{A} \\
\mathrm{B} \\
\mathrm{C} \\
\mathrm{D} \\
\mathrm{E} \\
\mathrm{B}\end{array}$ & $\begin{array}{l}5.11 \\
9.91 \\
9.91 \\
8.60 \\
5.11 \\
8.18 \\
7.81 \\
7.43\end{array}$ & $\begin{array}{l}12.80 \\
16.18 \\
16.18 \\
15.70 \\
12.80 \\
15.11 \\
15.06 \\
14.80\end{array}$ & $\begin{array}{r}\text { Percent } \\
100.0 \\
126.4 \\
126.4 \\
122.7 \\
100.0 \\
118.0 \\
117.7 \\
115.6\end{array}$ & $\begin{array}{r}l b / i h p-h r \\
0.452 \\
.595 \\
.558 \\
.545 \\
.458 \\
.514 \\
.514 \\
.525\end{array}$ & $\begin{array}{r}\text { Percent } \\
27.9 \\
36.4 \\
35.9 \\
34.8 \\
27.6 \\
34.4 \\
33.8 \\
32.6\end{array}$ & $\begin{array}{r}\text { Degrees } \\
47.5 \\
37.3 \\
34.7 \\
36.5 \\
45.0 \\
38.2 \\
37.5 \\
38.6\end{array}$ & $\begin{array}{r}\text { in. } \mathrm{Hg} \\
0.47 \\
.64 \\
.56 \\
.75 \\
.23 \\
.59 \\
.77 \\
.67\end{array}$ & $\begin{array}{r}\text { Degrees } \\
47.7 \\
36.3 \\
34.3 \\
34.7 \\
47.0 \\
37.5 \\
36.3 \\
36.6\end{array}$ \\
\hline
\end{tabular}

${ }_{1}$ Corrected to 0.50 in. $\mathrm{Hg}$ humidity, basis $7.3^{\circ}$ in. $\mathrm{Hg}$.

4 Nat. Advisory Comm. Rep. No. 426 (1932).

5 Nat. Adivsory Comm. Note No. 309 (1929).

6 SAE Journal 25, 277 (September 1929).

7 BS J. Research 3, 795 (1929) RP118.

8 SAE Journal 24, 155 (February 1929). 
The observed optimum spark advances and prevailing humidities are given in the seventh and eighth columns of table 18, and the advances corrected to a humidity of $0.50 \mathrm{in} . \mathrm{Hg}$, by the correction factor deduced above, in the last column of this table. These corrected advances vary, of course, with compression ratio. However, ethanol fuel, $\mathrm{C}$, and its ether blend, fuel $\mathrm{D}$, are somewhat below the mean line for the other fuels.

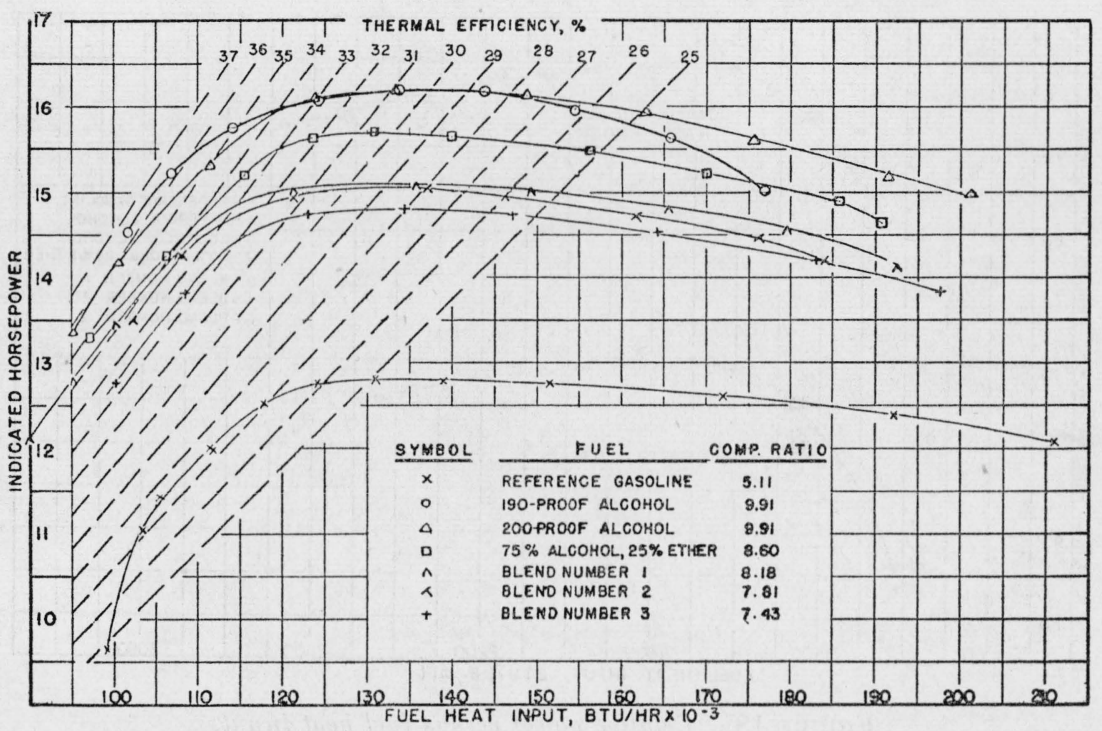

FiguRe 17.-Power and thermal efficiency of the test fuels at the compression ratio giving trace knock.

The peaks of the power curves occur at an essentially constant fuel heat input, although the compression ratios vary from 5.11 to 9.91 .

\section{COMBUSTION PERFORMANCE OF FUELS}

(a) RELATIVE POWER VERSUS HEAT INPUT

As a means of further comparing the combustion performance of the fuels, the data plotted in figures 16 and 17 have been transformed to percentages of maximum indicated power (for each fuel and test condition) and heat input in Btu per pound of dry air. The results are shown in figure 18 , in which the upper curve is fitted to the data obtained on the substitute fuels when operating at trace knock, and the lower curve is fitted to the data obtained on all fuels when operating at 5.11 compression ratio.

With two exceptions, it is apparent that the performances of these fuels are identical, within experimental error, at equal heat-input rates over the full range of mixture ratios. The performance of gasoline is slightly below the average at mixtures leaner than that giving maximum power, and slightly better at richer mixtures. The opposite is true of the 190-proof ethyl alcohol, which shows a marked deterioration in performance at rich mixtures. The behavior of the gasoline is consistent with the assumption that some of its less volatile components pass through the engine without being burned. 
In figure 19 the curves of figure 18 are compared. The differences are very slight and do not exceed the probable experimental error of air-flow measurement at the two conditions. If the measured air flow at 5.11 compression ratio were 1 percent low, for example, then the two curves are really coincident. The conclusion drawn is, that the relative performances of these fuels are essentially identical under both test conditions.

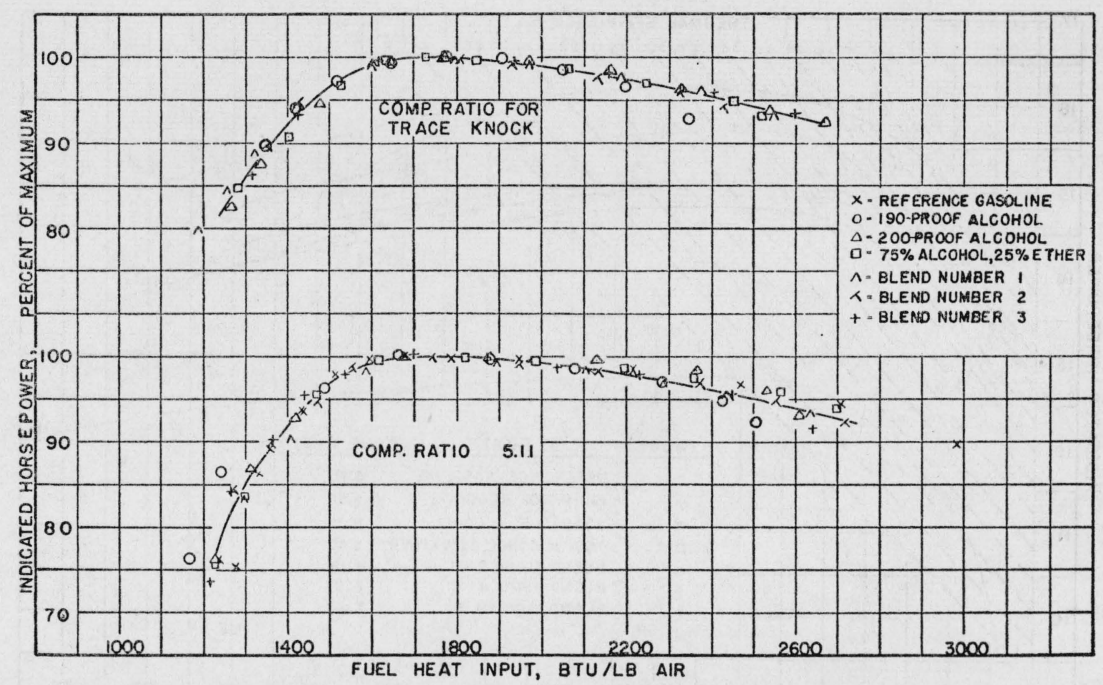

Figure 18.-Relative power versus fuel heat input.

When the power data of each curve on figures 16 and 17 are expressed as percentage of maximum power, the curves for each fuel coincide.

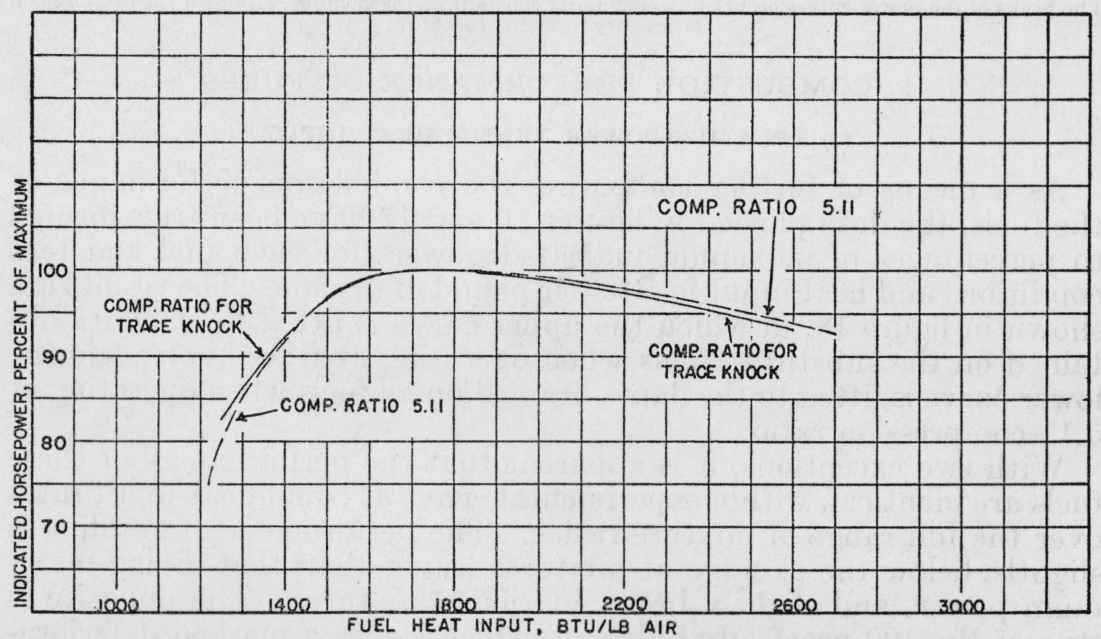

FIGURE 19.-Relative power versus fuel heat input.

The curves of figure 18 are shown by superposition to differ by no more than the experimental error. Thus hydrocarbon and nonhydrocarbon fuels, at compression ratios from 5.11 to 9.91 , show essentially the same response to mixture ratio. 
(b) RATE OF BURNING

Another means of comparing the combustion performance of the fuels is by a study of the combustion times as indicated by the optimum spark adyances. In figure 20, the optimum spark advances observed for each fuel at each mixture ratio when operating at a compression ratio of 5.11 have been corrected to a mean value of humidity of 0.50 in. $\mathrm{Hg}$ and plotted against fuel heat input in Btu per pound of dry air. Except at the leaner mixtures, the points do not show large scatter. The ether blend, fuel D, exhibits a definite tendency to require less advance than the other fuels. Gasoline appears to require a slightly higher advance than the other fuels at lean and at rich mixtures. It is noteworthy that, whereas maximum power is reached at 1,760 Btu per pound of air (fig. 18), the minimum advance, hence maximum combustion rate, occurs at 1,890 Btu per pound of air.

In order to compare the relation of optimum spark advance to mixture ratio at the lower and higher compression ratios used in the tests, the values shown in figure 20 and those obtained when operating at trace knock are plotted in figure 21 in percentage of minimum optimum spark advance. The curves of figure 21 are superposed in figure 22. This figure shows that at the higher compression ratios, the curve of relative optimum spark advance versus specific heat input is displaced in the direction of lower heat input, in this case by about 10 percent. This tendency is apparent also on closer examination of the upper plot on figure 21 , in which it can be noted that the points for fuels giving trace knock at the lower compression

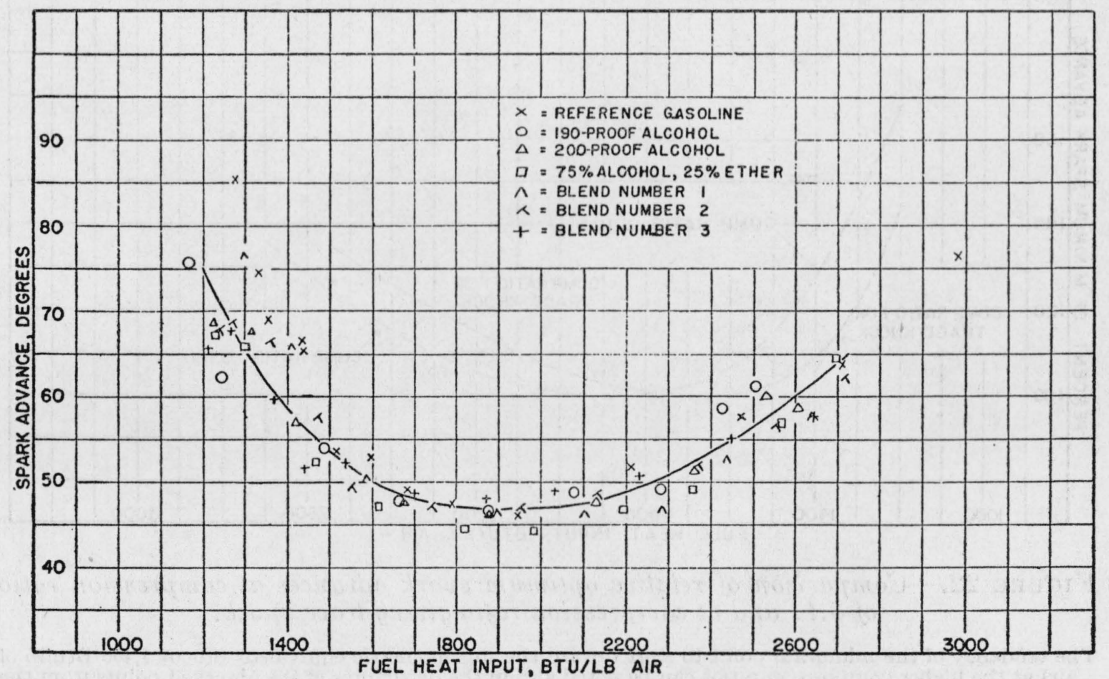

FIGURE 20.-Relation of optimum spark advance to fuel heat input.

In these tests at a compression ratio of 5.11, the observed optimum advances have been corrected to a standard pressure of water vapor (atmospheric humidity) of $0.50 \mathrm{in}$. Hg. Only the ether blend appears to differ substantially from the mean curve. 


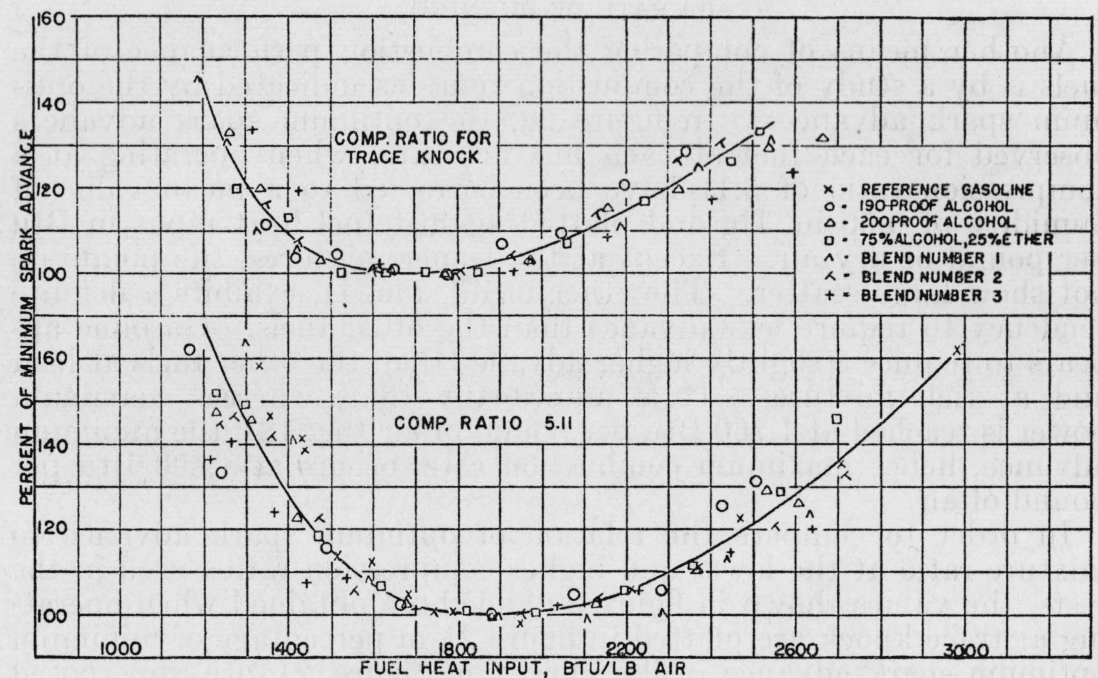

FIGURE 21.-Relative optimum spark advances.

When the optimum spark advances for each fuel are expressed in terms of the minimum optimum advance, the values are nearly coincident at the lower compression ratio, and in good agreement at the higher compression ratios.

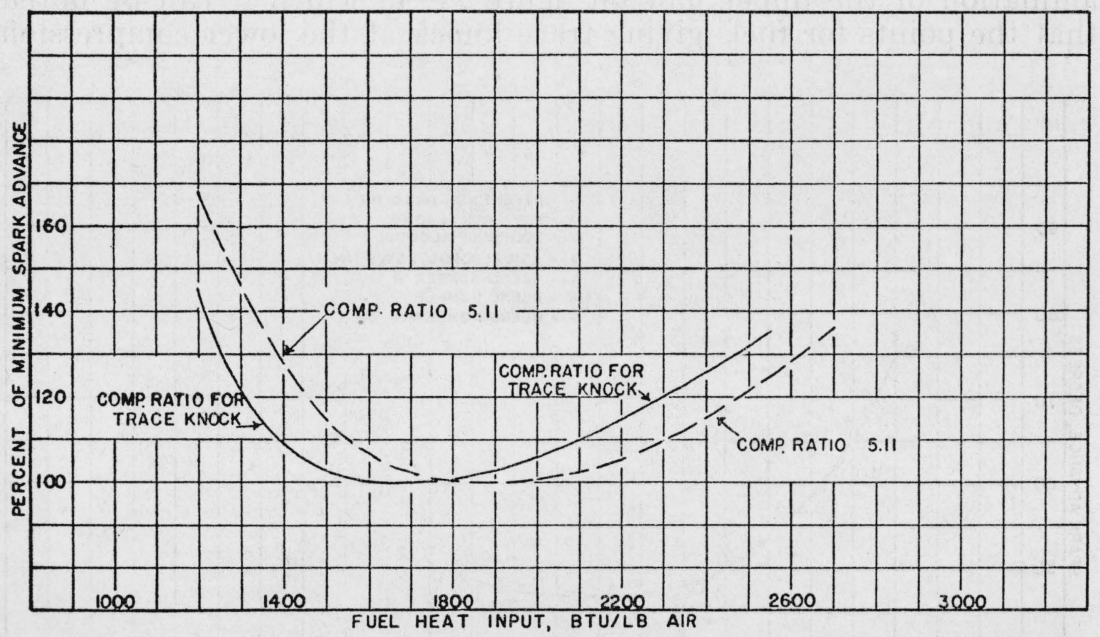

FIGURE 22.-Comparison of relative optimum spark advances at compression ratio of 5.11 and at compression ratio giving trace knock.

The tendency of the minimum point to shift toward the stoichiometric equivalent (about $1,400 \mathrm{Btu} / \mathrm{lb}$ of air) at the higher compression ratios can be noted also in the departures of the observed points from the upper curve of figure 21 .

ratios fall at higher fuel heat input than the curve, and vice versa. The significance of this fact can be expressed in several ways:

1. With increase of compression ratio, the actual optimum spark advance for lean mixtures decreases more rapidly than that for rich mixtures. 
2. With increase of compression ratio, the relative optimum spark advance for lean mixtures decreases and that for rich mixtures increases.

3. With increase of compression ratio, the mixture requiring minimum spark advance (presumably maximum rate of burning) approaches the stoichiometric equivalent.

The last statement follows from the fact that the heat input at the stoichiometric mixture ratio is roughly 1,400 Btu per pound of air for these fuels, as shown in table 17.

It is of interest to note that whereas at 5.11 compression ratio minimum optimum spark advance occurs at a mixture about 7 percent richer than that for maximum power, at the higher compression ratios used for trace knock with the substitute fuels it occurs at a mixture roughly 4 percent leaner than that for maximum power.

\section{(c) THERMAL-PLUG TEMPERATURES}

As mentioned earlier, the engine was equipped with a thermal plug mounted in the cylinder head to be flush with the combustionchamber surface. This plug was connected to a direct-reading indicator. Temperatures were noted at each test condition.

The readings obtained over the range of spark advance at each mixture ratio were plotted. The thermal plug temperature at optimum spark advance was then read from the resulting curve. Figure 23 shows these readings plotted against fuel heat input, a convenient basis for expressing mixture ratio in terms common to all fuels. Within experimental error, the observed temperatures for the various substitute fuels are equal, at comparable mixtures, both at 5.11 compression ratio and at trace knock. The temperatures observed for

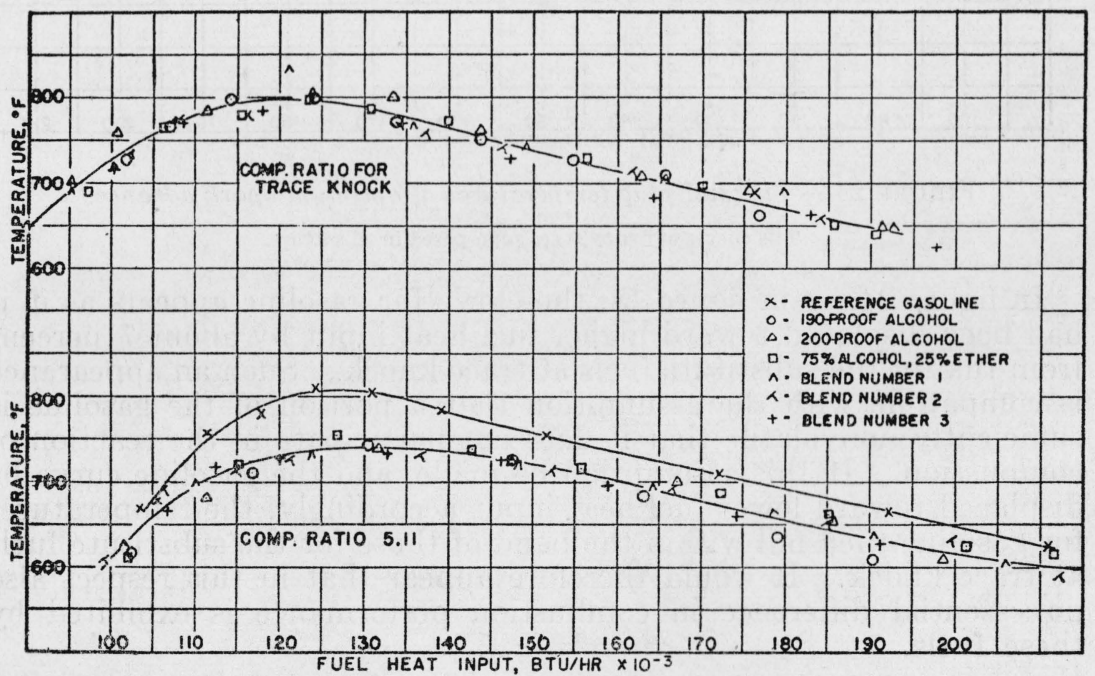

Figure 23.-Thermal plug temperatures at optimum spark advance.

The lowest curve is for the nonhydrocarbon fuels at a compression ratio of 5.11 , at which ratio these fuels did not knock. The curve immediately above is for gasoline, which gave trace knock at this ratio. The upper curve, on a different scale of ordinates, is for the nonhydrocarbon fuels at the compression ratio which gave trace knock with the fuel. 
gasoline, however, are decidedly higher than those for the substitute fuels at 5.11 compression ratio.

When the curves of this figure are superposed, as in figure 24 , it is seen that a considerable rise in temperature in the region lean of maximum power occurs when the substitute fuels are tested at trace knock instead of the lower compression ratio of 5.11. That most of this rise is connected with the phenomenon of knock and can be attributed to the occurrence of "incipient" knock is suggested by the fact that at richer mixtures, where no knock would be audible even at considerably higher compression ratios, the two curves for the substitute fuels differ by only about 12 degrees Fahrenheit, although the average compression ratio for trace knock for these fuels was 8.6 as compared with the value of 5.11 for the lower curve.

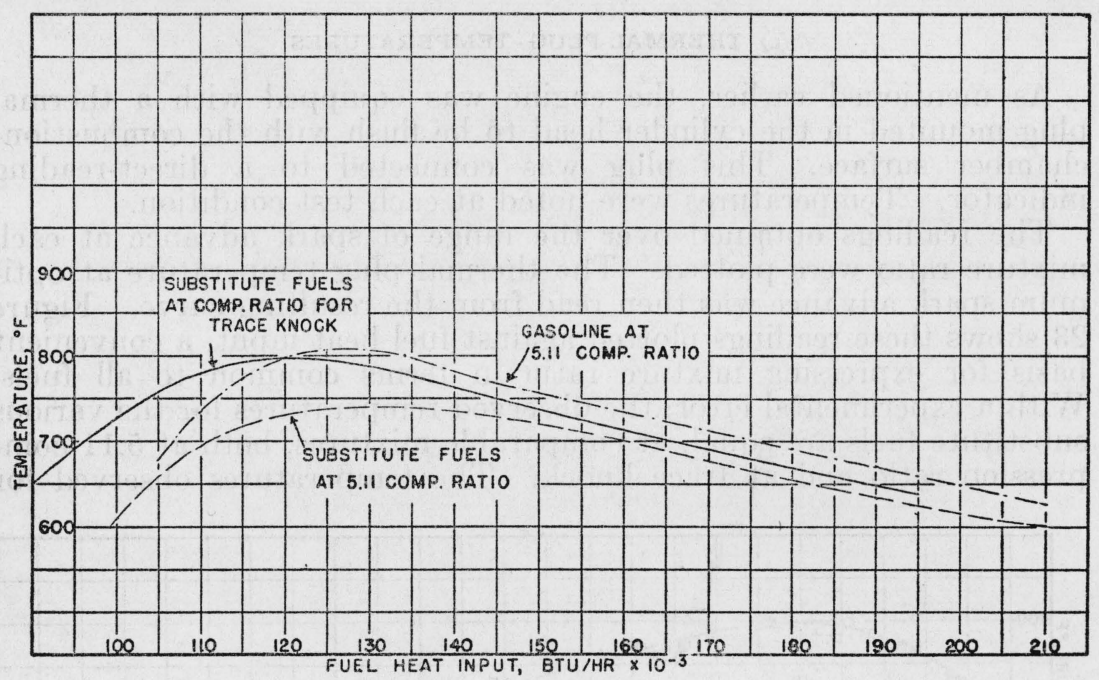

FIgURE 24.-Thermal plug temperatures at optimum spark advance.

The curves of figure 23 are superposed in this figure.

In figure 24, as in figure 18, the curve for gasoline appears as if it had been displaced toward higher fuel heat input by about 7 percent from that of the substitute fuels at trace knock. Such an appearance is compatible with the assumption that a portion of the gasoline is sufficiently nonvolatile that it does not participate in the reaction of combustion. If this assumption be made, and the gasoline curve be displaced toward lower fuel heat input accordingly, the temperatures for gasoline then fall within the band of those for the substitute fuels at trace knock. It would therefore appear that in this respect also no essential difference in combustion performance is exhibited by these fuels.

\section{POWER AT CONSTANT SPARK ADVANCE}

A plot of the data given in table 5 is shown for selected spark advances in figure 25, which is typical of power versus fuel-consumption curves at fixed spark advances. At the least-advanced spark, 40 
degrees, power is lower than with the other settings at lean and rich mixtures but approaches the best power obtained at the mixture ratio giving maximum power. At a setting of 50 degrees, power is considerably improved at lean and rich mixtures and is highest of any of the eurves at best mixture. An advance of 60 degrees improves the power at lean and rich mixtures, but causes a material loss of power in the intermediate range. The highest advance, 70 degrees, gives the highest power of any of these settings at the leanest mixture, and

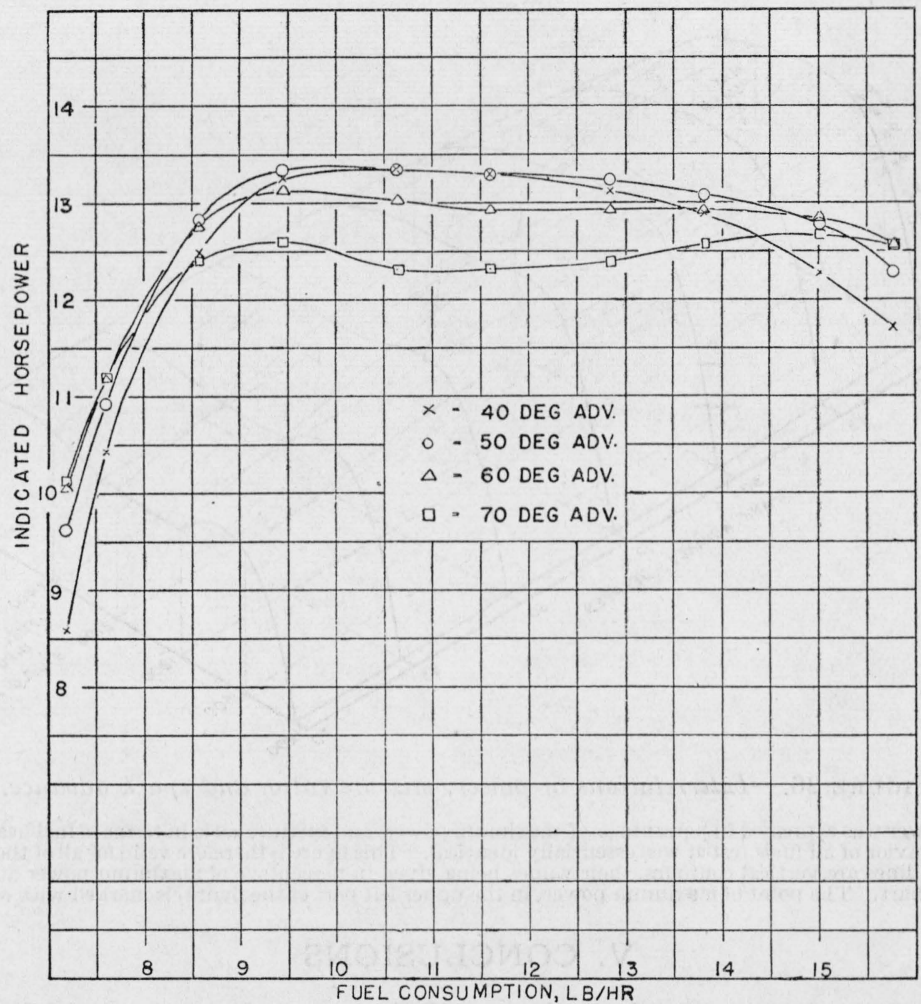

FIGURE 25.-Power curves at fixed spark advances.

Curves are shown for spark advances of $40^{\circ}, 50^{\circ}, 60^{\circ}$, and $70^{\circ}$. The reason for the double peak at $70^{\circ}$ is explained in the text.

would do so at a mixture slightly richer than any which were run, but gives a pronounced loss of power at normal mixtures.

The double peak shown by the power curve for 70-degree spark advance is typical of high spark advances. As can be seen in figures 1 to 14 , the optimum spark advance increases at lean and at rich mixtures. At high spark advances, the loss of power occasioned by going rich or lean from the best mixture is at first less than the gain of power resulting from the fact that at the richer or leaner setting the spark advance is closer to the optimum. This is the reason for the double peak of the power curve at high spark advances. Close examination of figure 25 will show that this double peak is foreshadowed in the curve for 60-degree advance. 


\section{POWER-MIXTURE-SPARK-ADVANCE RELATIONSHIP}

It was shown earlier in this analysis that when the data obtained on all fuels were plotted as percent maximum power versus fuel heat input in Btu per pound of air, the relations were essentially identical (figs. 18 and 19). The power-mixture-spark-advance surface for fuel D at 5.11 compression ratio, figure 26 , is therefore closely representative of that for all of these fuels, and the relations depicted therein may be considered as typical.

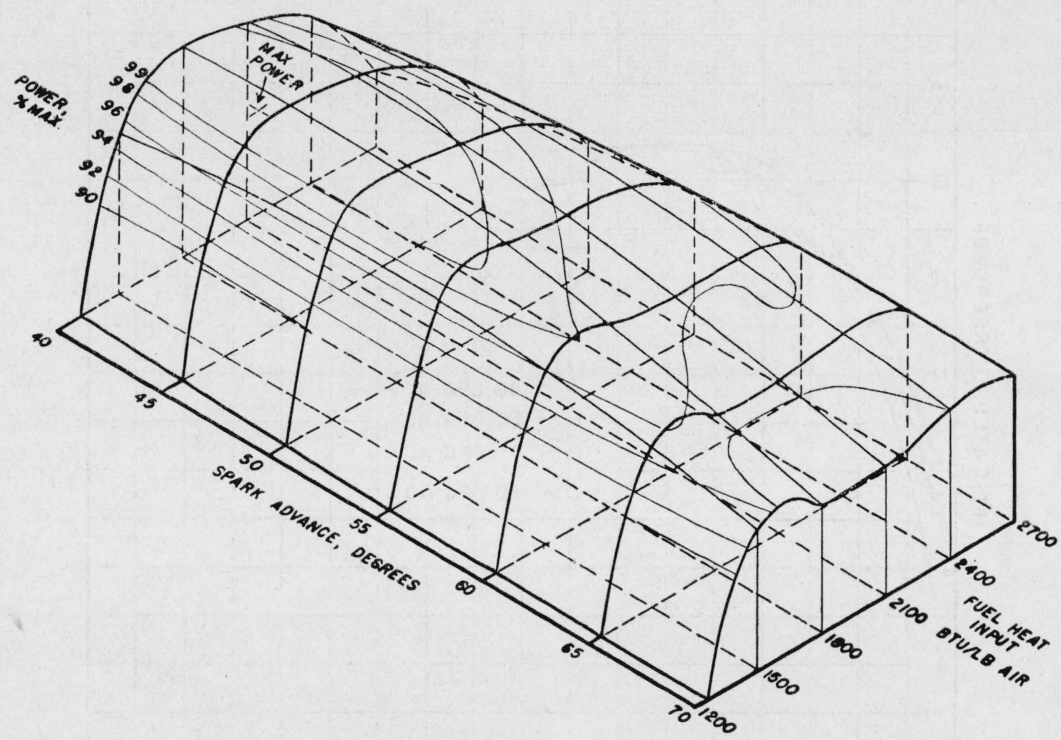

FIGURE 26.-Interrelations of power, mixture ratio, and spark advance.

When power was expressed in percentage of maximum power, and mixture ratio in terms of fuel heat input, the behavior of all fuels tested was essentially identical. This figure is therefore valid for all of these fuels. The light lines are vertical contours, their values being given in percentage of maximum power at the left of the chart. The point of maximum power, in the upper left part of the figure, is marked with a cross.

\section{CONCLUSIONS}

These tests on a single-cylinder CFR engine lead to the following conclusions, which are believed to be of general applicability in a qualitative sense:

1. At constant compression ratio, slightly more power (2 to 3 percent) is obtained with the acetone blends, whereas with 190 - or $200-$ proof ethyl alcohol the increase is somewhat larger (4 to 5 percent). At lean mixtures the substitute fuels have slightly better thermal efficiencies than does gasoline.

2. Analysis of the optimum spark advances of these fuels indicates that their rates of burning are equal at best mixture ratio, with the exception of the ether blend, which may burn a few percent faster.

3. All the substitute fuels are capable of use at compression ratios yielding more power than that allowable for the gasoline, with a corresponding increase in thermal efficiencies. The permissible increases in power range up to one-quarter for ethyl alcohol, the thermal efficiency of which is 36 percent, based on the higher heating value. 
4. Analyses of power, rate of burning as indicated by optimum spark advance, and thermal plug temperatures, versus fuel heat input, reveal no material differences in the combustion performances of these fuels.

5. Relative power at equal heat input per pound of air is the same for these fuels at both compression ratios used.

\section{APPENDIX}

\section{THERMAL EFFICIENCIES BASED ON LOWER HEATING VALUE}

The consensus of authorities in this country favors the use of the higher heating value of fuels in reckoning the thermal efficiency of an engine, although the lower heating value is preferred by some, and was standard practice in Germany. Although it may be considered improper to charge a noncondensing type of engine with the latent heat of vaporization of the water formed, it has been found that greater consistency of values results when the higher heating value of the fuels is used. For information, the thermal efficiencies of the several fuels, based on the lower heating values are given in table 19 .

TABLE 19.-Maximum thermal efficiency

[Based on lower heating value]

\begin{tabular}{|c|c|c|c|}
\hline \multirow[b]{2}{*}{ Fuel } & \multicolumn{2}{|c|}{ Thermal efficiency } & \multirow{2}{*}{$\begin{array}{l}\text { Compression } \\
\text { ratio for trace } \\
\text { knock }\end{array}$} \\
\hline & $\begin{array}{l}\text { At } 5.11, \text { com- } \\
\text { pression } \\
\text { ratio }\end{array}$ & $\begin{array}{l}\text { At compres- } \\
\text { sion ratio for } \\
\text { trace knock }\end{array}$ & \\
\hline $\begin{array}{l}\text { A } \\
\text { B } \\
\text { C } \\
\text { D } \\
\text { E } \\
1 \\
2\end{array}$ & $\begin{array}{c}\text { Percent } \\
29.9 \\
32.3 \\
31.8 \\
29.9 \\
29.5 \\
30.0 \\
31.1 \\
31.0\end{array}$ & $\begin{array}{c}\text { Percent } \\
29.9 \\
40.5 \\
39.6 \\
36.3 \\
29.5 \\
37.3 \\
36.7 \\
35.4\end{array}$ & $\begin{array}{c}\text { Percent } \\
5.11 \\
9.91 \\
9.91 \\
8.60 \\
5.11 \\
8.18 \\
7.81 \\
7.43\end{array}$ \\
\hline
\end{tabular}

Washington, March 21, 1945. 